\title{
Effects of epistasis and the evolution of genetic architecture: Exact results for a 2-locus model
}

\author{
José M. Álvarez-Castro ${ }^{\mathrm{a}, 1,2}$, Michael Kopp ${ }^{\mathrm{b}, 1}$, Joachim Hermisson ${ }^{\mathrm{b}, *}$ \\ a Swedish University of Agricultural Sciences, Department of Animal Breeding and Genetics, SE-75007 Uppsala, Sweden \\ ${ }^{\mathrm{b}}$ University of Vienna, Max F. Perutz Laboratories, Dr. Bohr-Gasse 9, A-1030 Vienna, Austria
}

\section{A R T I C L E I N F O}

\section{Article history:}

Received 29 July 2008

Available online 8 January 2009

\section{Keywords:}

Mutation-selection model

Epistasis

Genetic variance

Genetic architecture

Canalization

\begin{abstract}
A B S T R A C T
We study a two-locus model of a quantitative trait with a continuum-of alleles and multilinear epistasis that evolves under mutation, selection, and genetic drift. We derive analytical results based on the socalled House of Gauss approximation for the genetic variance, the mean phenotype, and the mutational variance in the balance of the evolutionary forces. The analytical work is complemented by extensive individual-based computer simulations. We find that (1) analytical results are accurate in a large parameter space; (2) epistasis always reduces the equilibrium genetic variance, as predicted in earlier studies that exclude drift; (3) large-scale stochastic fluctuations and non-equilibrium phenomena like adaptive inertia can strongly influence the evolution of the genetic architecture of the trait.
\end{abstract}

(c) 2009 Elsevier Inc. All rights reserved.

\section{Introduction}

Epistasis, the interaction among gene effects on phenotype, has traditionally played only a minor role in quantitative genetics. Indeed, the additive model of a quantitative trait, introduced by Fisher (1918) has dominated the literature in the last century. In this classical framework, epistasis only appears as a statistical correction term that can often be ignored in practical applications. In the last decade, however, the advent of molecular and statistical tools for the study of gene networks and quantitative trait loci (QTL) has started to change the picture. As the complex developmental or metabolic pathways connecting the genes that underlay a phenotypic trait were increasingly better understood, also the potential evolutionary importance of gene interactions was re-discovered as a research issue (Wolf et al., 2000).

Several new frameworks have been developed to include epistasis into genetic models, following two main strategies. On the one hand, new models in the context of QTL analysis extend the statistical approach to epistasis (Kao and Zeng, 2002; Yang, 2004; Zeng et al., 2005; Álvarez-Castro and Carlborg, 2007). On the other hand, the availability of estimates from real data made it clear that a conceptually different way of modeling called "physiological" or "functional" - is necessary, where model

\footnotetext{
* Corresponding author.

E-mail address: joachim.hermisson@univie.ac.at (J. Hermisson).

1 These authors have contributed equally to this article.

2 Current address: University of Santiago de Compostela, Department of Genetics, Avda Carvalho Calero s/n, 27002 Lugo, Spain.
}

parameters for epistasis correspond to the interactions between specific genes rather than to statistical averages (Cheverud and Routman, 1995; Hansen and Wagner, 2001; Rice, 2002; Barton and Turelli, 2004; Liberman and Feldman, 2006; Álvarez-Castro and Carlborg, 2007).

Various evolutionary questions are addressed with epistatic models. Part of these questions concern classical issues, such as the maintenance of genetic variation (Hermisson et al., 2003) or the predicted selection response (Carter et al., 2005), where the effect of epistasis can be studied relative to the traditional additive-effect model. In addition, there are new issues that can only be addressed in an epistatic model, like the evolution of the genetic architecture, i.e. the change of allelic effects at a focal locus due to changes in the genetic background (Hermisson et al., 2003; Hansen et al., 2006; Kopp and Hermisson, 2006; Liberman and Feldman, 2006), see also Hansen (2006) for a review. In this context, the evolution of small mutational effects is referred to as mutational robustness. The idea of a robust genetic architecture that is buffered against deleterious mutations picks up the classical theme of canalization (Waddington, 1953) and has seen a lot of recent research activity (reviewed in de Visser et al. (2003) and Flatt (2005)).

In this article, we present a comprehensive study of a minimal model of epistasis with two loci, which both allow for a continuum of alleles. We extend previous work on this system by Hermisson et al. (2003) in four directions. First, we present an improved analytical approximation for the genetic variance in mutationselection balance. The results are based on the recently developed "House of Gauss" approximation (Waxman, 2003; Hermisson and Wagner, 2004), which unifies and extends the classical House of 
Cards (Turelli, 1984) and Gaussian (Lande, 1976a) approximations that hold in complementary regions of the parameter space. We extend this approach to an epistatic model, where we can also derive predictions on the evolution of genetic architecture. As a second extension, we add genetic drift to the model. We derive analytical predictions using a stochastic version of the House of Gauss approximation and compare these to results from extensive individual-based simulations. Third, we study the effect of linkage among loci. Finally, we also discuss the transient behavior of the model, such as the time that is needed before an evolutionary equilibrium is reached. We find that selective constraints in the epistatic model can lead to adaptive inertia that substantially slows down the approach to the equilibrium points even in large populations.

\section{Model and analytical results}

The model follows Hermisson et al. (2003). We consider a quantitative trait in a randomly mating diploid population with equivalent sexes. Ignoring gene-environment interactions, the genotypic value is given by the following map

$x=x_{\mathrm{opt}}+y_{1}+y_{2}+\varepsilon y_{1} y_{2}$.

$x_{\mathrm{opt}}$ is the optimal trait value, which we choose as our reference. Neglecting dominance, the single-locus variables $y_{i}=a_{i}+a_{i}^{*}$ are determined additively by the maternal and paternal effects. We allow for a continuum of alleles at both loci, $a_{i}, a_{i}^{*} \in \mathbb{R}$. Also the epistasis parameter $\varepsilon$ is arbitrary, but is constrained to the same value for all alleles at the interacting loci. The model is thus a (simple) case of the multilinear epistasis model by Hansen and Wagner (2001).

Mutation at the $i$ th locus occurs at a diploid (twice the haploid) rate $u_{i}$ and adds a random increment $\delta_{i}$ to the single-locus variable $y_{i}$. The distribution $\rho\left(\delta_{i}\right)$ of $\delta_{i}$ is assumed to be symmetric with mean zero and variance $\gamma_{i}^{2}$. We assume weak stabilizing selection with a quadratic fitness function,

$w(x)=1-s\left(x-x_{\mathrm{opt}}\right)^{2}$

To avoid negative fitness values, we restrict the phenotype space to $\left|x-x_{\text {opt }}\right| \leq 1 / \sqrt{s}$. Note that we are not interested in an absolute value for the trait (which is arbitrary), but rather its distance from the optimal phenotype, $\left|x-x_{\text {opt }}\right|$. By an appropriate choice of a reference point for trait measurements, and a rescaling of the model parameters $\gamma_{i}^{2}$ and $\varepsilon$, we can always set $x_{\text {opt }}=0$ (cf. Hansen and Wagner (2001) and Hermisson et al. (2003)). In this case, $|x|$ is a direct measure for this distance. Since the model with $x_{\text {opt }}=0$ is symmetric under the mapping $y_{1,2} \rightarrow-y_{1,2}, x \rightarrow-x$, and $\varepsilon \rightarrow-\varepsilon$, we can focus on positive $\varepsilon \geq 0$.

Following Hermisson et al. (2003), we introduce the epistasis factor for the $i$ th locus $(i=1,2)$ as

$f_{i}:=\frac{\partial}{\partial y_{i}} x=1+\varepsilon y_{3-i}$.

With epistasis, mutational effects at each locus depend on the genetic background. This background-effect is captured by the epistasis factor. The effect of a mutation of size $\delta_{i}$ on the phenotype $x$ is $\delta_{i} f_{i}$. The average selection coefficient of a mutation at locus $i$ is (to leading order in $s$ )

$$
\begin{aligned}
& \int\left(w(x)-w\left(x+\delta_{i} f_{i}\right)\right) \rho\left(\delta_{i}\right) \mathrm{d} \delta_{i} \\
& =-s \int\left(x^{2}-\left(x+\delta_{i} f_{i}\right)^{2}\right) \rho\left(\delta_{i}\right) \mathrm{d} \delta_{i} \\
& =s \gamma_{i}^{2} f_{i}^{2} .
\end{aligned}
$$

Epistasis factors can be represented in vector form as

$$
\boldsymbol{f}=\nabla x=\mathbf{1}+\mathbf{E} \boldsymbol{y}
$$

where $\boldsymbol{y}$ is the vector with components $y_{1}$ and $y_{2}, \mathbf{1}$ is the unit vector, $\nabla x$ denotes the gradient of $x$ with respect to the locus variables, and $\mathbf{E}$ is the epistasis matrix (Rice, 1998)

$\mathbf{E}=\left(\begin{array}{cc}0 & \varepsilon \\ \varepsilon & 0\end{array}\right), \quad E_{i j}=\frac{\partial^{2} x}{\partial y_{i} \partial y_{j}}$.

We denote population averages (over a single generation) by angled parentheses $\langle\ldots\rangle$, but we will also use the over-bar as abbreviation for single letters, i.e. $\left\langle a_{i}\right\rangle=\bar{a}_{i}$. It will be convenient to calculate with whole-locus (diploid) quantities. Due to equivalence of the sexes, and assuming Hardy-Weinberg proportions throughout, the whole-locus cumulants are just twice the corresponding haploid ones. In particular, we have $\bar{y}_{i}=2 \bar{a}_{i}$ and $V_{i}=2 \operatorname{Var}\left[a_{i}\right]$ for the means and variances of the locus reference effects $y_{i}$. In the stochastic version of the model, we denote averages over replicates by $E[\cdot]$.

The statistical properties of the trait and of fitness can be expressed in terms of the epistasis factors. Assuming linkage equilibrium, the following relations have been established in Hermisson et al. (2003):

$\bar{x}=\frac{1}{2}\left(\overline{\boldsymbol{f}}^{t} \mathbf{E}^{-1} \overline{\boldsymbol{f}}-\mathbf{1}^{t} \mathbf{E}^{-1} \mathbf{1}\right)=\left(\bar{f}_{1} \bar{f}_{2}-1\right) / \varepsilon$

where $f^{t}$ denotes the transpose and $\mathbf{E}^{-1}$ is the inverse epistasis matrix. The genetic variance $V_{G}$ can be decomposed into an additive $\left(V_{A}\right)$ and epistatic $\left(V_{A A}\right)$ part, which read

$V_{G}=V_{A}+V_{A A}=\bar{f}_{1}^{2} V_{1}+\bar{f}_{2}^{2} V_{2}+\varepsilon^{2} V_{1} V_{2}$,

and the mutational variance is given by

$V_{m}=u_{1} \gamma_{1}^{2}\left\langle f_{1}^{2}\right\rangle+u_{2} \gamma_{2}^{2}\left\langle f_{2}^{2}\right\rangle$,

where $(i=1,2)$

$\left\langle f_{i}^{2}\right\rangle=\bar{f}_{i}^{2}+\varepsilon^{2} V_{3-i}$

is the mean squared epistasis factor, which measures the epistatic effect of the genetic background on variance components. The mutation load $L$ can be expressed in terms of the phenotypic mean and variance as $L=s\left(\bar{x}^{2}+V_{G}\right)$. In mutation-selection balance, the variance in fitness equals the negative population mean effect of mutation on fitness. For a multilinear trait and quadratic stabilizing selection, we therefore obtain

$$
\begin{aligned}
\operatorname{Var}[w] & =-s \sum_{i=1,2} u_{i} \int\left(\left\langle\left(x+\delta_{i} f_{i}\right)^{2}\right\rangle-\left\langle x^{2}\right\rangle\right) \mathrm{d} \delta_{i} \\
& =-s \sum_{i=1,2} u_{i} \int\left(2 \delta_{i}\left\langle x f_{i}\right\rangle-\delta_{i}^{2}\left\langle f_{i}^{2}\right\rangle\right) \mathrm{d} \delta_{i} \\
& =s \sum_{i=1,2} u_{i} \gamma_{i}^{2}\left\langle f_{i}^{2}\right\rangle=s V_{m}
\end{aligned}
$$

where we use that the average mutational effect is zero. We thus find that the variance in fitness is proportional to the mutational variance of the trait.

The per-generation change of the means and variances of the single locus reference effects, $\bar{y}_{i}$ and $V_{i}$, is twice the change of the corresponding haploid quantities, which is given by the Price equations. We assume linkage equilibrium, weak mutation and selection, and an infinite population size. The whole-locus changes then read

$$
\begin{aligned}
& \Delta \bar{y}_{i}=2 \Delta \bar{a}_{i}=2 \operatorname{Cov}\left(a_{i}, w\right), \\
& \Delta V_{i}=2 \Delta \operatorname{Var}\left[a_{i}\right]=2 \operatorname{Cov}\left(\left(a_{i}-\bar{a}_{i}\right)^{2}, w\right)+u_{i} \gamma_{i}^{2} .
\end{aligned}
$$


From (14), the following relations for the change of the means and the variances of the locus reference effects may be derived (Turelli and Barton, 1990; Hermisson et al., 2003),

$$
\begin{aligned}
\Delta \bar{y}_{i} & =V_{i} \frac{\partial}{\partial \bar{y}_{i}} \bar{w}+C_{3, i} \frac{\partial}{\partial V_{i}} \bar{w} \\
& =-2 s V_{i}\left(\bar{x} \bar{f}_{i}+\sum_{j \neq i} \varepsilon_{i j} V_{j} \bar{f}_{j}\right)-s C_{3, i}\left\langle f_{i}^{2}\right\rangle, \\
\Delta V_{i} & =\left(V_{i}^{2}+C_{4, i}\right) \frac{\partial}{\partial V_{i}} \bar{w}+C_{3, i} \frac{\partial}{\partial \bar{y}_{i}} \bar{w}+u_{i} \gamma_{i}^{2} \\
& =-s\left(V_{i}^{2}+C_{4, i}\right)\left\langle f_{i}^{2}\right\rangle-2 s C_{3, i}\left(\bar{x} \bar{f}_{i}+\sum_{j \neq i} \varepsilon_{i j} V_{j} \bar{f}_{j}\right)+u_{i} \gamma_{i}^{2} .
\end{aligned}
$$

For the two-locus model, $\varepsilon_{i j}=\varepsilon$ and the sums run only over a single term. $C_{3}$ and $C_{4}$ denote the third and fourth cumulant of the distribution of $y_{i}$. In equilibrium, $C_{3}$ vanishes due to symmetry and the dynamic equation for the means reduces to an eigenvalue equation (Hermisson et al., 2003)

$\mathbf{E V} \overline{\boldsymbol{f}}=-\bar{x} \overline{\boldsymbol{f}}$,

where the variance matrix $\mathbf{V}$ is diagonal (due to linkage equilibrium), and the elements on the diagonal are the locus components $V_{1}, V_{2}$. There are two standard approximations for the fourth order cumulant. In the Gaussian approximation (Lande, 1976b), a normal distribution for the allelic values at all loci is assumed, and thus $C_{4}=0$. Predictions from the Gaussian approximation are valid in the limit of small mutational effects and large mutation rates, where one can show that $V^{2} \gg C_{4}$ (Bürger, 2000). The opposite limit with few mutations of large effect defines the domain of the House-of-Cards (HC) approximation (Turelli, 1984). Here, we can set $V_{i}^{2} \approx 0$ and approximate the fourth cumulant as $C_{4, i}=V_{i} \gamma_{i}^{2}$.

In Hermisson et al. (2003), results based on the Gaussian and HC approximations were given. A simple framework that extends these approximations and works for the whole parameter range has recently been suggested by Waxman (2003) and Hermisson and Wagner (2004). In the so-called House-of-Gauss (HG) approximation (Hermisson and Wagner, 2004), we follow the HC approximation and set $C_{4, i}=V_{i} \gamma_{i}^{2}$, but we do not ignore $V_{i}^{2}$. For a single locus, the $\mathrm{HG}$ framework reproduces a functional form for the equilibrium genetic variance that was first derived by Waxman (2003) as the exact solution of a continuum-of-alleles model with a special shape of the mutational-effect distribution,

$\rho(\delta)=\frac{\delta}{\gamma^{2} \sinh \left[\pi \delta / \sqrt{2 \gamma^{2}}\right]}$.

This distribution is symmetric with variance $\gamma^{2}$ and kurtosis 1 and does not deviate much from a Gaussian in its appearance. For a general distribution of mutational effects, the HG scheme works as a convenient approximation that can also be extended to include epistasis. In particular, we find for our model

$\Delta V_{i}=-s\left(V_{i}^{2}+V_{i} \gamma_{i}^{2}\right)\left\langle f_{i}^{2}\right\rangle+u_{i} \gamma_{i}^{2}$.

If we define $g_{i}:=s \gamma_{i}^{2}$, this has the equilibrium solution (for $\Delta V_{i}=$ $0)$

$V_{i}=\frac{\gamma_{i}^{2}}{2}\left(\sqrt{1+4 u_{i} /\left(g_{i}\left\langle f_{i}^{2}\right\rangle\right)}-1\right)$

In the absence of epistasis ( $\varepsilon=0$ and $f_{i}=1$ ), Eq. (4) shows that $g_{i}$ is the average selection coefficient. With epistasis, selection is background dependent and the average marginal effect on fitness of a mutation at the $i$ th locus becomes $g_{i}\left\langle f_{i}^{2}\right\rangle$, which enters (21).

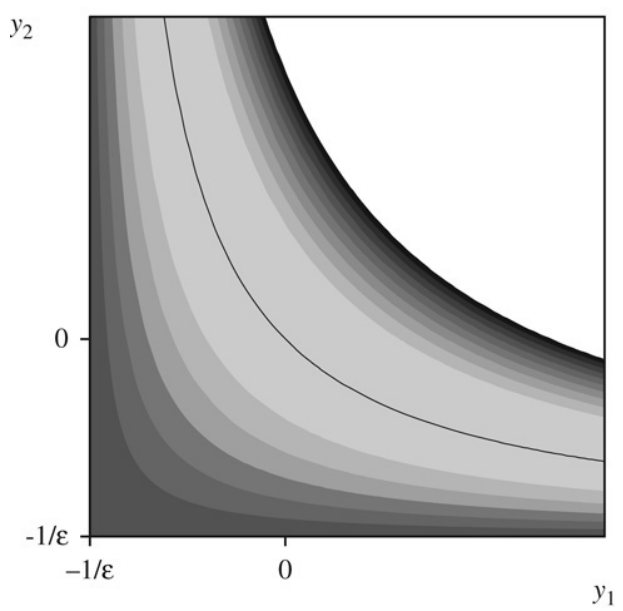

Fig. 1. Graphical representation of the fitness landscape (i.e., the map from the locus effects $y_{1}$ and $y_{2}$ to fitness) in the presence of epistasis. The solid line corresponds to the optimal phenotype. Darker colors signify decreasing fitness. Note that the landscape is symmetric with respect to the diagonal $y_{1}=y_{2}$.

To determine the equilibrium values of $\left\langle f_{i}^{2}\right\rangle$, we need to employ the equilibrium condition for the locus means. From Eq. (18), we obtain $f_{1}^{2} / f_{2}^{2}=V_{2} / V_{1}$ and

$\bar{x}=-\varepsilon \sqrt{V_{1} V_{2}}$

where condition (7) determines the negative sign of $\bar{x}$. From (7), (10) and (18) we then also obtain, for $\varepsilon \neq 0$,

$\left\langle f_{1}^{2}\right\rangle=\sqrt{V_{2} / V_{1}}=\left\langle f_{2}^{2}\right\rangle^{-1}$.

Eqs. (22) and (23) fix the position of the population on the phenotype- (and fitness-) landscape. Fig. 1 shows that this landscape forms a hyperbolic ridge of high fitness around the contour of the optimal phenotype $x=0$. According to Eq. (22), the equilibrium mean phenotype deviates slightly from this optimum, in the direction of the flatter slope of the ridge. Movement of the population along the ridge changes the locus effects in opposite directions. Consequently, Eq. (23) shows that a large scaling factor $\left\langle f_{1}^{2}\right\rangle$ for the first locus in equilibrium corresponds to a small scaling factor $\left\langle f_{2}^{2}\right\rangle$ at the second locus, and vice versa. As we discuss below, movement of the population along the ridge can be interpreted as evolution of the genetic architecture.

We can use (23) to eliminate the epistasis factors from the equations for $V_{1,2}$ (Eq. (21)). To this end, it is convenient to define a variable

$z:=\frac{\gamma_{2}}{\gamma_{1}} \sqrt{\frac{V_{1}}{V_{2}}}=\sqrt{\frac{g_{2} V_{1}}{g_{1} V_{2}}}$.

We then obtain from Eqs. (21) and (23)

$V_{1}=\frac{\gamma_{1}^{2} z}{2 \sqrt{g_{1} g_{2}}}\left(\sqrt{g_{1} g_{2} z^{-2}+4 u_{1} \sqrt{g_{1} g_{2}} z^{-1}}-\sqrt{g_{1} g_{2}} z^{-1}\right)$

and for $V_{2}$ the same equation with $\gamma_{1}^{2}$ and $u_{1}$ replaced by $\gamma_{2}^{2}$ and $u_{2}$, and $z$ replaced by $z^{-1}$. From these two expressions, and using Eq. (24), we can express $\sqrt{V_{1} V_{2}}$ in two ways as

$$
\begin{aligned}
\sqrt{V_{1} V_{2}} & =\frac{1}{2 s}\left(\sqrt{g_{1} g_{2} z^{-2}+4 u_{1} \sqrt{g_{1} g_{2}} z^{-1}}-\sqrt{g_{1} g_{2}} z^{-1}\right) \\
& =\frac{1}{2 s}\left(\sqrt{g_{1} g_{2} z^{2}+4 u_{2} \sqrt{g_{1} g_{2}} z}-\sqrt{g_{1} g_{2}} z\right) .
\end{aligned}
$$

Equating (26) and (27), we obtain after some transformations

$\left(u_{1} z^{-1}-u_{2} z\right)^{2}-\sqrt{g_{1} g_{2}}\left(u_{1}-u_{2}\right)\left(z-z^{-1}\right)=0$. 
Table 1

Analytical predictions for the variance $V$ of the single-locus reference effects $y_{i}$ in the case of identical loci (i.e., equal mutation rates and mutational variances; $u_{1}=$ $\left.u_{2}=u, \gamma_{1}^{2}=\gamma_{2}^{2}=\gamma^{2}\right) . \Theta=4 N_{e} u$ and $G=2 N_{e} s \gamma^{2}$. The mean phenotype is given by $\bar{x}=-\varepsilon V$ and the total genetic variance by $V_{G}=2 V-\varepsilon^{2} V^{2}$. Setting $\varepsilon=0$ gives the equations for the additive case. (S)G: (Stochastic) Gaussian approximation (S)HC: (Stochastic) House-of-Cards approximation; (S)HG: (Stochastic) House-ofGauss approximation. The stochastic versions are derived in the Appendix.

\begin{tabular}{llll}
\hline$G$ & $\gamma \sqrt{\frac{u}{s}}$ & SG & $\frac{\sqrt{1+2 \Theta G}-1}{4 N_{e} s}$ \\
HC & $\frac{u}{s}$ & SHC & $\frac{2 N_{e} u \gamma^{2}}{G+1}$ \\
HG & $\frac{\gamma^{2}}{2}\left(\sqrt{1+\frac{4 u}{s \gamma^{2}}}-1\right)$ & SHG & $\frac{\gamma^{2}}{2 G}\left(\sqrt{G^{2}+2 G(1+\Theta)+1}-G-1\right)$ \\
\hline
\end{tabular}

This is a fourth order equation in $z$, which can be solved analytically with Mathematica, but the solution is usually a lengthy expression and therefore not shown here. In the Gaussian limit, where $u_{i} \gg g_{i}$, we find $z=z_{G}=\sqrt{u_{1} / u_{2}}$ for $u_{1} \geq u_{2}$. For the full system, the equilibrium is $z \geq z_{G}$. In fact, the HC solution formally corresponds to $z=\infty$ if $u_{1} \neq u_{2}$, (cf. Hermisson et al., 2003). For equal mutation rates, $u_{1}=u_{2}$, we see from Eq. (28) that $z=1$ is the unique solution.

It is possible to include genetic drift into our formalism. If $N_{e}$ is the diploid effective population size, this leads to an additional term $-V_{i} / 2 N_{e}$ in the dynamical equation for the variances (20) since drift reduces genetic variation. Explicit formulas and all derivations are given in the Appendix. As in the House-of-Gauss (HG) case (21), we obtain a "Stochastic House-of-Gauss" (SHG) approximation for the variance components (A.2) as a function of the average squared epistasis factor. An analogous calculation as for infinite population size again leads to a quartic equation for the parameter $z$.

From the solution for $z$ in the infinite or finite population size case, predictions for the equilibrium values of all statistical quantities follow easily. In particular, we obtain the locus components for the variances from Eq. (25), and all epistasis factors from Eqs. (10) and (23). One may note that the equilibrium values for $z, V_{i}$ and the epistasis factors are all independent of the interaction parameter $\varepsilon$, given that $\varepsilon \neq 0$. This can be understood as follows. Epistasis, in this model, relaxes the constraint of the additive model, where both locus effects are fixed. Evolution uses this additional degree of freedom to drive the loci to an equilibrium where the single-locus quantities only depend on the model parameters $u_{i}, \gamma_{i}^{2}$, and $s$ (for smaller $\varepsilon$, the population evolves further out on the fitness ridge). Also the equilibrium mutational variance is a function of $z$,

$V_{m}=u_{1} \gamma_{1}^{2}\left\langle f_{1}^{2}\right\rangle+u_{2} \gamma_{2}^{2}\left\langle f_{2}^{2}\right\rangle=\gamma_{1} \gamma_{2}\left(u_{1} z^{-1}+u_{2} z\right)$,

and therefore independent of $\varepsilon$. In contrast, the phenotype mean and the equilibrium genetic variance depend explicitly on $\varepsilon$, see Eqs. (8) and (24). For equal loci (equal mutation rates and mutational variances), we obtain simple explicit expressions for all equilibrium values. Table 1 summarizes the key results from the HG and SHG formalism in comparison with the deterministic and stochastic versions of the previous Gaussian (G) and House-ofCards (HC) approximations.

In all calculations above, complete linkage equilibrium among the loci was assumed. This is generally a good approximation if recombination is much stronger than selection. For the additive model, even moderate levels of linkage have little effect on the equilibrium quantities (cf. Bürger, 2000, p. 248ff). Since linkage disequilibria can result from epistasis, however, this is far less clear if there are epistatic interactions among loci. In order to study the effects of weak linkage, we can follow the standard approach and calculate the leading order correction terms to the linkage equilibrium solution. We measure linkage disequilibrium between the two loci by the correlation of their allelic values,
$\rho_{12}=C_{12} / \sqrt{V_{1} V_{2}}$, where $C_{12}=\operatorname{Cov}\left[y_{1}, y_{2}\right]$ is the covariance. If $r$ is the recombination rate, the change among generations of $C_{12}$ is $\Delta C_{12}=-r C_{12}+\Delta_{s} C_{12}$. Even for a simple two-locus model, the selection response $\Delta_{S} C_{12}$ is a complicated expression that involves multiple products of higher-order cumulants. In the quasi-linkage-equilibrium approximation (Turelli and Barton, 1990), the selection response is approximated by its linkage equilibrium counterpart, $\Delta_{\mathrm{LE}} C_{12}$, which can take a much simpler form. We focus on the deterministic model. The selection response for $C_{12}$ at mutation-selection balance of the epistatic model assuming linkage-equilibrium then reads (compare Eq. (17))

$$
\begin{aligned}
\Delta_{\mathrm{LE}} C_{12} & =\left\langle\frac{\delta w}{\delta y_{1} \delta y_{2}}\right\rangle_{\mathrm{LE}} V_{1} V_{2} \\
& =-s\left(\bar{f}_{1} \bar{f}_{2}+\varepsilon \bar{x}\right) V_{1} V_{2} \\
& =-s(1+2 \varepsilon \bar{x}) V_{1} V_{2},
\end{aligned}
$$

where we make use of the fact that all cross-locus cumulants and all cumulants of order three vanish at this point. The quasi-linkage equilibrium approximation for the correlation follows as

$\rho_{12} \approx \frac{\Delta_{\mathrm{LE}} C}{r \sqrt{V_{1} V_{2}}}=-s(1+2 \varepsilon \bar{x}) \frac{\sqrt{V_{1} V_{2}}}{r}$.

As expected, this value is negative for the additive model $(\varepsilon=0)$ and also for moderate epistasis. Interestingly, since $\bar{x}=$ $-\varepsilon \sqrt{V_{1} V_{2}}+\mathcal{O}[C]<0$, linkage disequilibrium is reduced by epistasis on the level of the trait. This can be understood by noting that linkage disequilibria result from epistasis for fitness, which is always present under stabilizing selection, even for the additive model. In our model, fitness epistasis is measured by the derivative $\varepsilon_{w}:=\partial w /\left(\partial y_{1} \partial y_{2}\right)$. Eq. (30) shows that $\varepsilon_{w}=-s\left(1-2 \varepsilon^{2} \sqrt{V_{1} V_{2}}\right)$, i.e. epistasis on the phenotypic level reduces (the absolute value of) fitness epistasis. This can be understood as a consequence of the slight shift of the population mean in the direction of the more shallow slope of the fitness landscape.

\section{Simulation results}

To test the validity and accuracy of the analytical approximations, we performed stochastic individual-based computer simulations. In the simulations, populations consisted of $N$ individuals, with each individual being defined by a set of diploid two-locus genetic effects (i.e., allelic values). For each generation, we first computed the phenotypes of all the individuals, based on their genetic effects, using (1). Second, the fitnesses were computed using (2). Third, $N$ pairs of individuals were selected for reproduction, using their fitnesses as weights and assuming equal numbers of males and females. Each selected pair produced one offspring, accounting for recombination and mutation. Mutations occurred at rate $u_{i}(i=1,2)$ per individual locus, and their additive effects were taken from a normal distribution with mean zero and variance $\gamma_{1}=\gamma_{2}=\gamma^{2}$.

The above procedure ensures that the effective population size $N_{e}$ equals the actual population size $N$, because mating pairs are sampled randomly and each pair has exactly one offspring. Thus, the number of offspring per individual is identical to the number of matings it participates in, which follows an (approximate) Poisson distribution with mean and variance $2 . N_{e}$ then follows from Eq. (E4) in Bürger (2000, page 363).

Simulations were run in the following way: In the initial populations, all reference effects were set to zero, unless otherwise stated. For each parameter combination, we ran 10 replicate simulations with 1200,000 generations for cases with equal locus parameters $\left(u_{1}=u_{2}\right.$ and $\left.\gamma_{1}^{2}=\gamma_{2}^{2}\right)$, or 3000,000 generations 

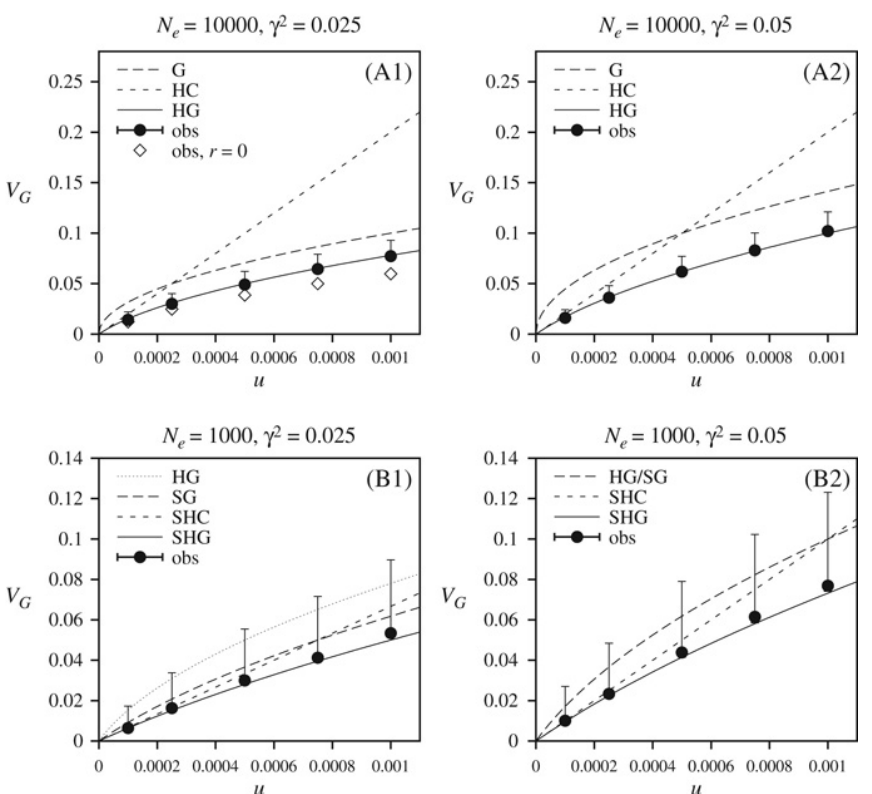

Fig. 2. Genetic variance $V_{G}$ as a function of the per locus mutation rate $u$ in the additive model $(\varepsilon=0)$ for both large $\left(N_{e}=10,000\right)$ and small $\left(N_{e}=1000\right)$ populations and for two different values of $\gamma^{2}$, the variance of the mutational effects. In (A1), we also show the mean genetic variance in a model with complete linkage $(r=0)$. Results are averages over 10 simulation runs (see main text). Upward error bars show the average standard deviation over time. The standard deviation of the replicate means (downward error bars) is too small to be visible at this scale. Lines show the predictions from the various deterministic and/or stochastic approximations. In (B2), the deterministic HG coincides with the stochastic $\mathrm{G}$ (this is because $\left.1 /\left(2 N_{e} s\right)=\gamma^{2}\right)$.

for cases with unequal locus parameters. From these simulations, we sampled relevant statistics every 1000 generations, starting at generation 200,000 (equal loci) or 1000,000 (unequal loci), when most populations had reached a state of equilibrium (exceptions are pointed out in the text). The sampled data were then used for further analysis. In all cases shown, the strength of selection was set to $s=0.01$.

\subsection{Equilibrium mean phenotype and genetic variance}

As reference for the effect of epistasis, we start with a brief evaluation of the (S)HG approximation in the additive model. Fig. 2 $\mathrm{A} 1$ and $\mathrm{A} 2$ show the results and predictions for the equilibrium genetic variance $V_{G}$ in a large population $\left(N_{e}=10,000\right)$. The HG predictions fit the simulations very well. In particular, $\mathrm{HG}$ provides much better predictions than the previous approximations (HC and $\mathrm{G})$ for the whole parameter range. We use two types of error bars in Fig. 2 (as well as in Figs. 3 and 8). Upward error bars indicate the standard deviation across generations. They thus mark the range where we can expect to find the population in mutation-selectiondrift equilibrium. Downward error bars measure the standard deviation of replicate means and thus the precision of our point estimates. In Fig. 2, these error bars are too small to be perceived (but see e.g. Fig. 3D3). In the additive model, the predicted mean phenotype always coincides with the optimum, $\bar{x}=0$, and the simulation results are very close to this value (not shown).

Fig. 2B1 and B2 show the effect of a small population size $\left(N_{e}=\right.$ 1000) and compares the SHG approximation to the stochastic versions of the previous Gaussian and HC approximation. The improvement is similar to the one for a large population, although slight deviations of the simulation results from the predictions are now visible. Comparison with the deterministic HG approximation shows the impact of drift on the average value of $V_{G}$ across generations. The upward error bars, which cover also the deterministic approximation, indicate a considerably larger standard deviation than for $N_{e}=10,000$.

Fig. 3 shows the effect of epistasis on the equilibrium values of $\bar{x}$, $V_{G}$ and $V_{m}$. For the phenotypic mean, the analytical theory predicts a slight deviation in the negative direction, that is, in the direction where the slope of the fitness ridge is shallower (see also Rice, 1998). Fig. 3A1 and B1 show that the HG predictions fit the simulation results very well for large population sizes. According to the analytical results, epistasis leads to a slight reduction of the equilibrium genetic variance by a term $\sim \varepsilon^{2} V^{2}$. This is confirmed by simulations. In particular, Fig. $3 \mathrm{~A} 2$ and $\mathrm{B} 2$ show that the HG prediction for large populations are again very precise. Additional results for a larger range of the epistasis parameter $\varepsilon$ are presented in Fig. A.1 the Appendix. Note that the upward error bars for the standard deviation among generations are usually larger than the predicted epistasis effect, for both $\bar{x}$ and $V_{G}$, even for $N_{e}=10,000$.

In rows $C$ and $D$ of Fig. 3 the same results are shown for a small population $\left(N_{e}=1000\right)$. Genetic drift plays a much greater role with epistasis than without epistasis, as evidenced by the large standard deviations (both among replicate means and across generations). Note also that, for small population sizes, the SHG prediction is considerably less accurate with epistasis than without epistasis (compare Fig. 3C2 and D2 to Fig. 2B1 and B2). This happens because the SHG approximation only accounts for averages among replicates, but ignores the variances. In particular, for equal loci, the approximation is consistent with the population being at the symmetric point of its optimal contour (with equal reference effects, $y_{1}=y_{2}$ ), where the genetic variance is minimized. Simulated and real populations will instead fluctuate along the iso-phenotype contour, reaching areas where the slope of the phenotype landscape is steeper, and the genetic variance larger. A similar effect does not occur in the additive model since, in this case, the phenotype (and fitness) landscape is translationally invariant along the contour $y_{1}+y_{2}=0$. The better fit of the stochastic Gaussian (SG) approximation for some parameter values (Fig. $3 \mathrm{C} 2$ ) is a result of fortuitous cancelation of error terms.

The right-most column of Fig. 3 shows the mutational variance $V_{m}$. The prediction from the HG approximation is $V_{m}=2 u \gamma^{2}$ (for both the deterministic and stochastic versions). The observed values are consistently above this estimate. The reason is again that the approximations neglect stochastic fluctuations of the population around the average equilibrium position. Fluctuations occur mostly along the iso-phenotype contour of $\bar{x}$ (compare Fig. 9 below for the case of unequal loci). In contrast to $\bar{x}$, the epistasis factors $\left\langle f_{i}^{2}\right\rangle$ are very variable on this contour. According to Eq. (23), fluctuations in $\left\langle f_{1}^{2}\right\rangle$ and $\left\langle f_{2}^{2}\right\rangle$ are negatively correlated. For equal loci (and using Eq. (23)) we can write the mutational variance as $V_{m}=u \gamma^{2}\left(\left\langle f_{1}^{2}\right\rangle+\left\langle f_{1}^{2}\right\rangle^{-1}\right)$. Fluctuations thus have a non-linear effect on $V_{m}$ and introduce a bias. We can (partly) account for this effect by using the Taylor expansion $E\left[\left\langle f_{1}^{2}\right\rangle^{-1}\right] \approx\left(1 / E\left[\left\langle f_{1}^{2}\right\rangle\right]\right)+$ $\left(\operatorname{Var}\left[\left\langle f_{1}^{2}\right\rangle\right]\right) /\left(E\left[\left\langle f_{1}^{2}\right\rangle\right]\right)^{3}$ to obtain "corrected" values for $V_{m}$

$\tilde{V}_{m}=V_{m}-u \gamma^{2} \frac{\operatorname{Var}\left[\left\langle f_{1}^{2}\right\rangle\right]}{\left(E\left[\left\langle f_{1}^{2}\right\rangle\right]\right)^{3}}$

(open circles in Fig. 3) which fit the prediction more closely, at least as long as the deviations are not too big (for $N=10,000$ ). Note that these "corrected" values are not a better version of $V_{m}$, but merely demonstrate why the observed $V_{m}$ is typically greater than $2 u \gamma^{2}$.

Finally, Figs. 4 and 5 show the effects of linkage on the equilibrium quantities. From Fig. 4 , we see that (negative) linkage disequilibrium is indeed weaker in the equilibrium of the epistatic model than in the additive model. Consequently, the effect of linkage on the equilibrium genetic variance and the phenotype mean is very small. Effects are stronger for high mutation rates, but generally only visible for tight linkage $(r \lesssim 0.001$, Fig. 5$)$. Results for complete linkage $(r=0)$ are also added to Figs. 2 and 3 . Note the much smaller effect of linkage on the genetic variance $V_{G}$ in the epistatic case. 

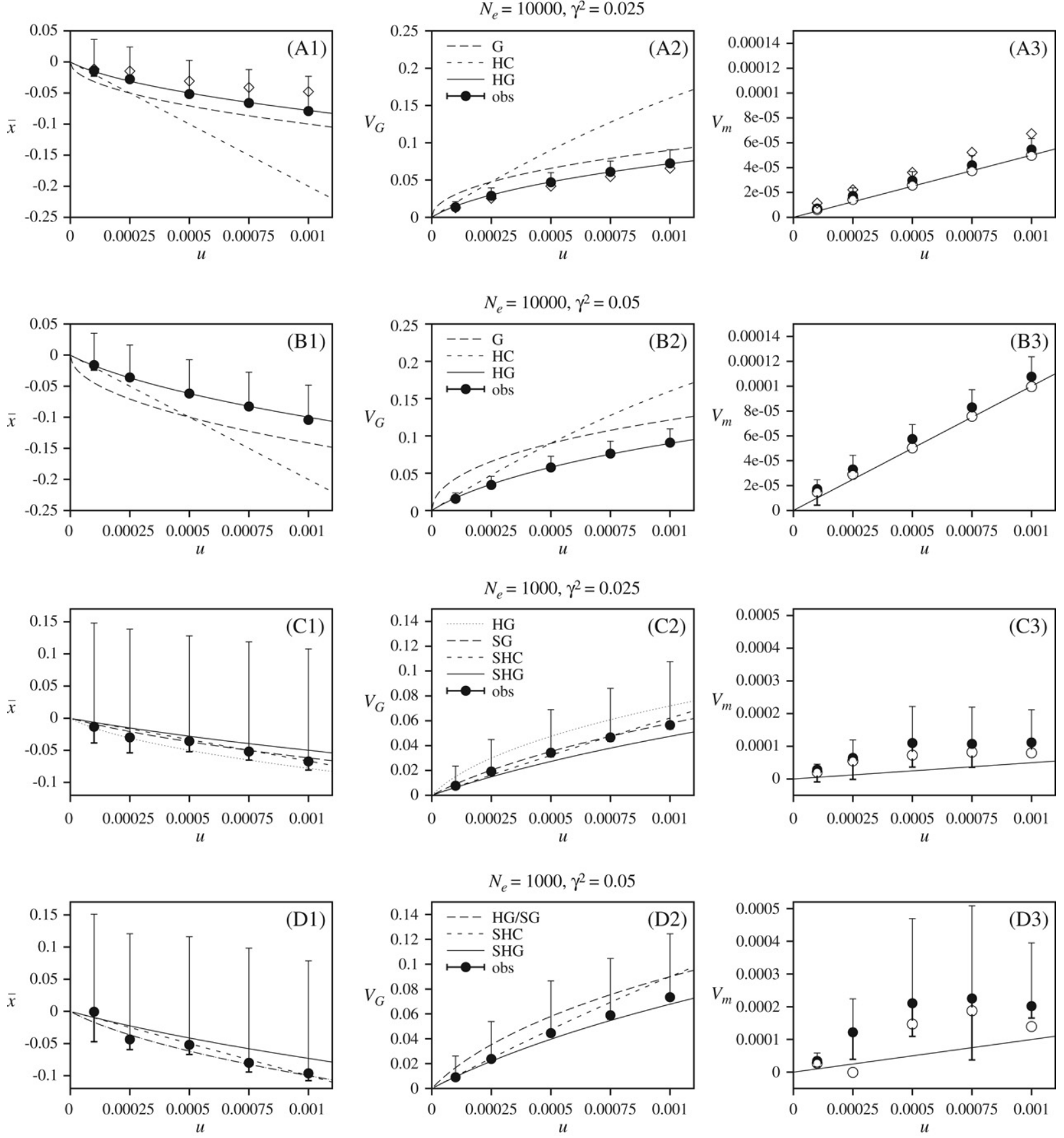

Fig. 3. Simulation results for the model with identical loci and epistasis. The plots show the phenotypic mean $\bar{x}$, the genetic variance $V_{G}$, and the mutational variance $V_{m}$ as a function of the per locus mutation rate $u$ in large $\left(N_{e}=10,000\right)$ and small $\left(N_{e}=1000\right)$ populations for $\varepsilon=2$ and two different values of $\gamma^{2}$. Upper error bars show mean standard deviations over time, whereas lower error bars, if visible, show the standard deviation over the 10 replicate means (see Fig. 2 for further details). In the first row, diamonds show the mean observed values in a model with complete linkage ( $r=0$, cf. Fig. 5). In the right-most column, the open circles show the means of the "corrected" mutational variance $\tilde{V}_{m}$ as defined in Eq. (32) (see text).

\subsection{The evolution of genetic architecture under epistatic selection}

For an additive trait, optimization of the mean phenotypic trait value is the sole target of selection. Consequently, the population simply evolves uphill the fitness landscape until the mean phenotype matches the optimum. In contrast, there is a second evolutionary target on an epistatic landscape. Selection aims to buffer deleterious alleles that segregate in the population (Rice, 1998; Hermisson et al., 2003). The consequences can be seen in Fig. 6, i.e. there is selection for the reduction of the genetic variance $V_{G}$. Since selection for the optimization of the mean phenotype is generally stronger, a population that starts far off the equilibrium will first evolve toward and actually reach the equilibrium phenotype contour. With epistasis, this one is close to - but not the same as - the optimal phenotype. In a second phase, selection for buffering of deleterious alleles in the standing genetic variation then moves the population along the contour. For equal mutation rates, the equilibrium is the symmetric point of the iso-phenotype contour, which is usually quickly reached. For unequal mutation rates, selection drives the population into one of the branches of the high-fitness ridge. Locus effects evolve in opposite directions: whereas the locus with the higher mutation rate is buffered and evolves small mutational effects, mutational effects at the locus with the lower mutation rate increase. 

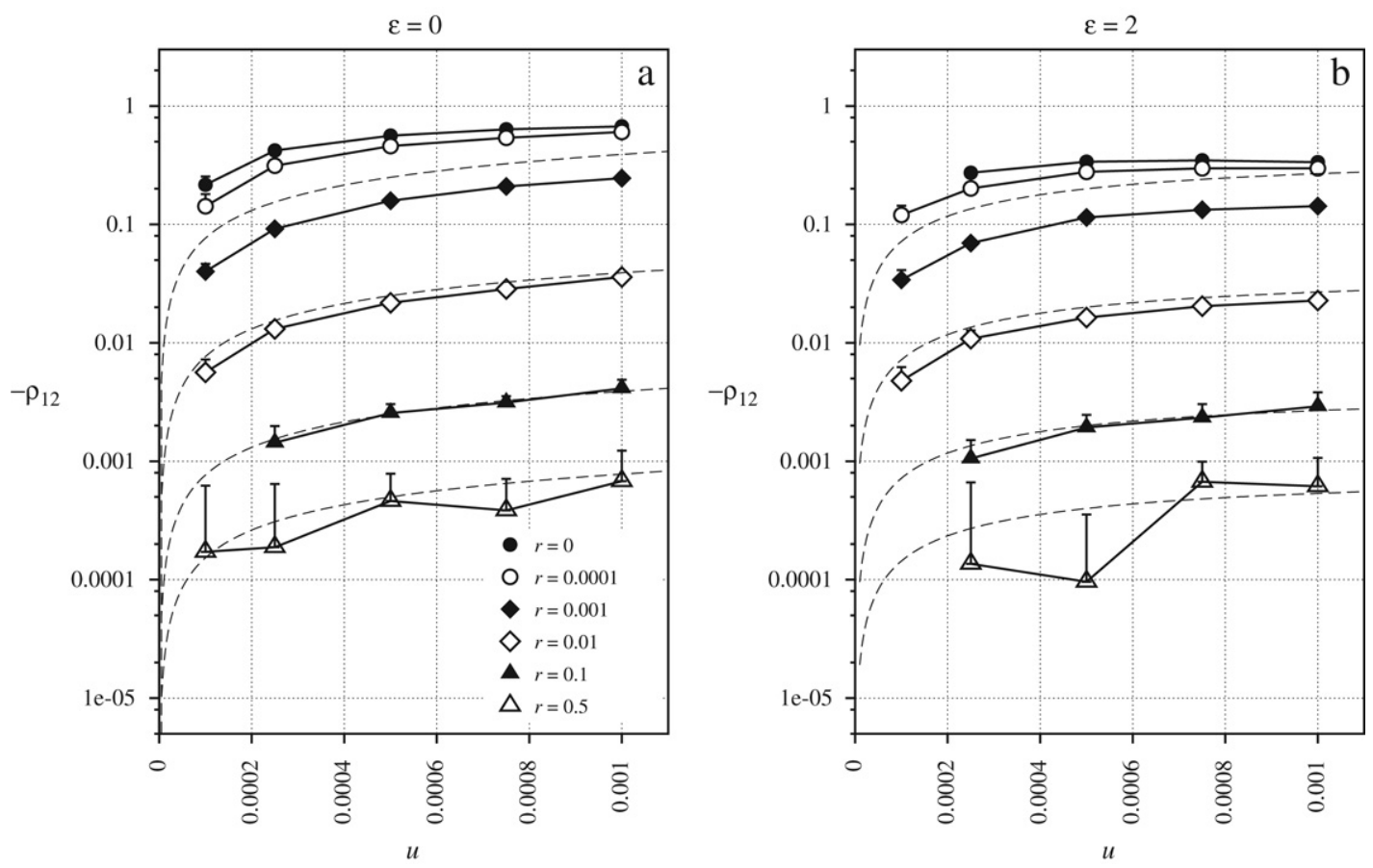

Fig. 4. Linkage disequilibrium in the model with equal loci, measured as the correlation $\rho_{12}$ between $y_{1}$ and $y_{2}$, the additive effects of the two loci. (a) Additive model $(\varepsilon=0)$, (b) model with epistasis $(\varepsilon=2)$. Other parameters were $\gamma^{2}=0.025$ and $N_{e}=10,000$. Note that all correlations are negative, but are shown here on a positive logarithmic scale. Unlike in Figs. 2, 3 and 8, error bars only show the standard deviation of replicate means (corresponding to lower error bars in the above figures). The dotted lines are the predictions from the quasi-linkage equilibrium approximation (31) for (from bottom to top) $r=0.5, r=0.1, r=0.01$, and $r=0.001$ (for smaller $r$, the approximation cannot be applied).

In order to compare simulation results for the evolution of genetic architecture with the analytical predictions, we introduce a variable transformation for the phenotype landscape. In the original coordinate system, spanned by the mean locus effects $\bar{y}_{1}$ and $\bar{y}_{2}$, evolution (in the extended second phase) occurs along a curved contour. This poses a problem when coordinates $\bar{y}_{i}$ are averaged over time and/or replicates: even if all individual data points are on the contour, the average might deviate from it. To solve this problem, we describe the position of a population on the landscape by its mean phenotype $\bar{x}$ (which fixes the iso-phenotype contour) and by its distance (arc-length) $\alpha$ along this contour from the main diagonal (see Fig. 7). Averages can then be calculated on this scale, before being back-transformed to the $\bar{y}_{1}, \bar{y}_{2}$ coordinate system. Formal transformation rules are given in the Appendix.

Fig. 8A-D shows the mean phenotypes, arc lengths, and the genetic and mutational variances in simulations of a large population with different values of $\gamma^{2}$ and $\varepsilon$. Significant deviations from the HG approximation occur only if the per-locus mutation rates are very different. In this case, the population needs to evolve a large distance along the iso-phenotype contour (compare Fig. 7B). While $\bar{x}$ and $V_{G}$ have (almost) converged to the predicted equilibrium values, the population does not reach the predicted arc length $\alpha$ within 3000,000 generations (see Fig. 9A2). Although the mutational variance $V_{m}$ is determined by the position of the population on the epistatic landscape (in particular by the arc length $\alpha$ ), the simulation results deviate more strongly from the analytical predictions. The reason is that $V_{m}$, in contrast to $\alpha$, depends non-linearly on fluctuations of the population along the iso-phenotype contour. As explained above for the case of equal loci, this introduces an upward shift of the simulated data. As in the case of equal locus effects, there is hardly any effect of moderate linkage $r \geq 0.001$ on the results. For comparison, results for complete linkage are added in Fig. 8A. Smaller population size leads to a large increase of all standard errors (Fig. 8E). As in the case of equal loci, the SHG approximation slightly under-estimates the average $V_{G}$ (see Fig. 8E3).
Fig. 9 shows the evolutionary trajectories of typical example populations for different parameter values. We observe that evolution along the iso-phenotype contour is very slow, but punctuated by larger jumps. Slow evolution is not due to weak selection relative to genetic drift: increase in population size does not strongly affect the speed of evolution (see Fig. 9A1-A3). The reason is rather that evolution on a narrow ridge of the epistatic landscape is only possible if fine-tuned mutations at both loci contribute. Epistasis thus creates "adaptive inertia" due to the need of correlated evolution (Baatz and Wagner, 1997, see discussion below). Population size mainly affects the frequency of the stochastic jumps along the contour. These occur when a slightly deleterious mutation at the second locus (with smaller mutation rate) fixes due to drift and is then compensated by a large change at the first locus (with higher mutation rate). Stochastic jumps and adaptive inertia can combine to large-scale fluctuations of the population with extended excursions (up to $10^{6}$ generations in Fig. 9B2) far away from the predicted equilibrium values.

A central prediction of Hermisson et al. (2003) for the evolution of the genetic architecture was that canalization (buffering of mutational effects) evolves for the locus with the higher mutation rate, while the locus with the smaller mutation rate is decanalized. In particular, the contribution to the mutational variance of a locus with a higher mutation rate is predicted to be smaller than the contribution of the locus with lower mutation rate. This is indeed found in all simulations with unequal mutation rate, even in the presence of evolutionary inertia (Fig. 8A4-E4, open and closed triangles). An example for the evolution of the per-locus mutational variances is shown in Fig. 10a. Starting at a symmetric point on the phenotype landscape, $V_{m}$ is initially dominated by the locus with the higher mutation rate. As the genetic architecture evolves, a crossing of the locus contributions occurs after on the order of $10^{4}-10^{5}$ generations, depending on the mutation rates, see Fig. 10b. 

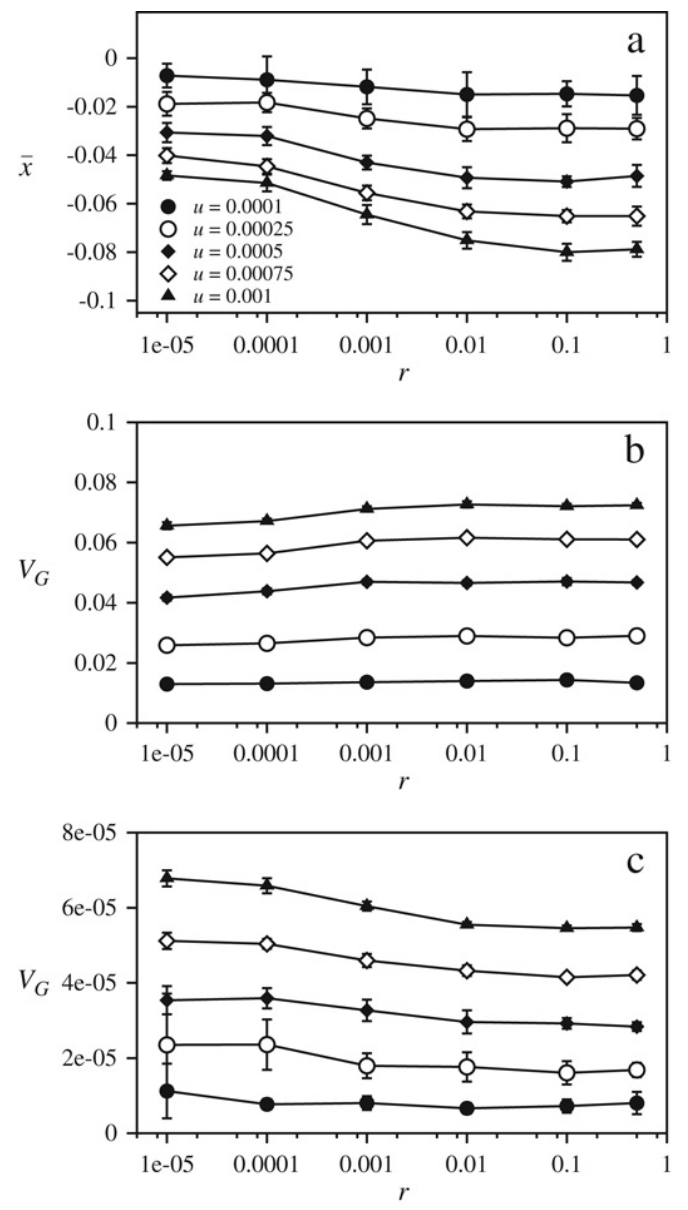

Fig. 5. The effect of linkage in the model with identical loci and epistasis. The plots show the phenotypic mean $\bar{x}(\mathrm{a})$, the genetic variance $V_{G}(\mathrm{~b})$, and the mutational variance $V_{m}$ (c) as a function of the recombination rate $r$, for various values of the per-locus mutation rate $u$ in a large population $\left(N_{e}=10,000\right)$ for $\varepsilon=2$ and $\gamma^{2}=0.025$ (cf., Fig. 3A1-A3). Values for $r=0$ (which cannot be shown at this scale) are very similar to those for $r=10^{-5}$. Unlike in Figs. 2, 3 and 8, error bars only show the standard deviation of replicate means (corresponding to lower error bars in the above figures).

\section{Summary and Discussion}

\subsection{The house of Gauss approximation}

Our simulations show that in the absence of epistasis both the HG and the SHG approximations provide highly accurate predictions for the genetic variance in mutation-selection (and drift) balance (Fig. 2). This result holds for basically the entire parameter space of biological interest, from small to large mutation rates and/or locus effects. In contrast, validity of the classical HC and $G$ approximations (and their stochastic variants) are each restricted to complementary subsets of the parameter space. While the $G$ is adequate when mutation rates are high or mutational effects are low, the HC covers the cases where mutations are rare and have relatively big effects (see Bürger (2000)). In the additive case, HG and SHG consistently out-perform the better one of the previous approximations. The largest relative improvement is seen at the border that separates the $\mathrm{HC}$ and the $\mathrm{G}$ regimes, i.e. for $u / s \approx \gamma^{2}$.

With epistasis, the HG approximation constitutes a clear improvement over the combination of $\mathrm{HC}$ and $\mathrm{G}$ approximation that was used in Hermisson et al. (2003). For large populations $\left(N_{e} \geq 10,000\right)$, the HG values for the mean phenotype and the equilibrium genetic variance are very accurate (Fig. 3). Also the predicted position on the phenotype ridge (arc length $\alpha$ ) fits well to the asymptotic long-term averages in the simulation runs (Fig. 8). For small populations, a consistent deviation of the SHG approximation from the numerical values appears that is not seen in the additive case. The reason is that epistasis leads to an extended variance of the population-averaged observables $\left(V_{G}, V_{m}, \bar{x}\right)$ among replicates and across generations. This variance is neglected in all stochastic approximations. For the mutational variance $V_{m}$, which has a strong non-linear dependence on stochastic fluctuations of the population around the equilibrium points (i.e., along the contour), deviations are even visible for larger populations.

The limitations of the HG approximation, and of any analytical point estimate of evolutionary observables in mutation-selectiondrift balance, are most obvious for the epistatic model with unequal locus mutation rates. The simulation results show extended stochastic excursions of the population away from the predicted equilibrium points (Fig. 9). As explained above, large-scale fluctuations may lead to shifts in the averages across generations and replicates. More fundamentally, with excursions lasting for tens of thousands of generations, equilibrium predictions by simple point estimators are only of limited value (see also the discussion of adaptive inertia below). a

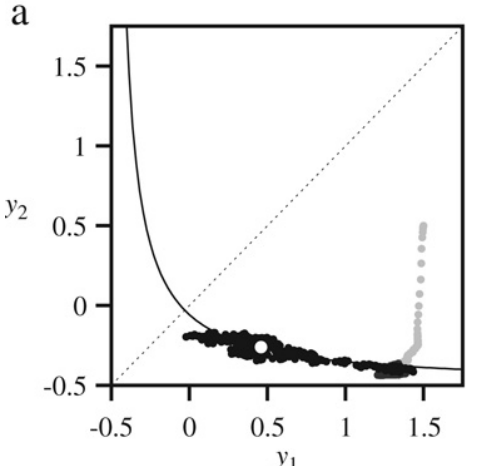

b

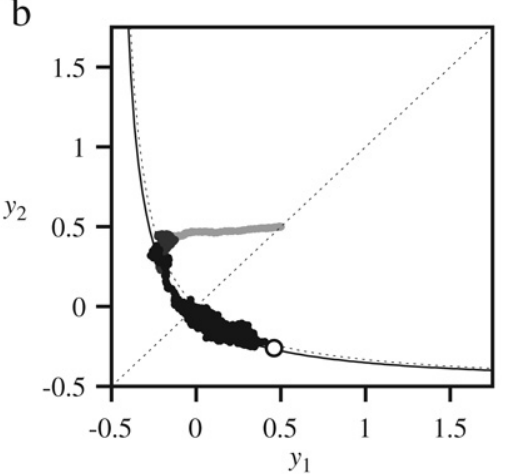

c

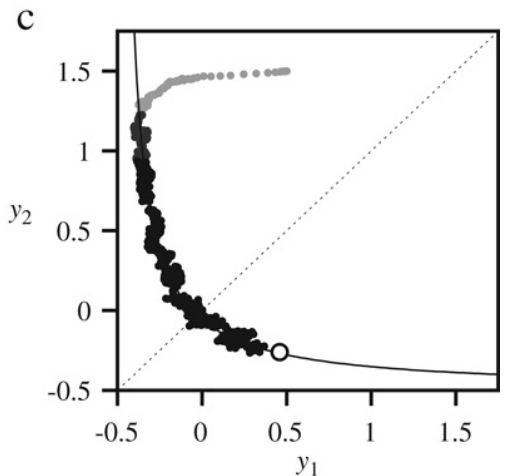

Fig. 6. Approach of the equilibrium phenotype contour from different initial conditions. The plots show trajectories for the evolution of the locus reference effects $y_{1}$ and $y_{2}$ from three different starting values. Light grey: Generations 0-1000; dark grey: generations 1000-5000; black: generations 5000-100,000. The solid line is the equilibrium contour and the open circle the predicted equilibrium point. The dotted line is the main diagonal. In (b), the additional dotted line shows the contour of the optimal phenotype $x=0$. Samples were taken every 10 generations for the first 5000 generations and every 100 generations for subsequent generations. Starting values: (a) $y_{1}=1.5, y_{2}=0.5$; (b) $y_{1}=0.5, y_{2}=0.5$; (c) $y_{1}=0.5, y_{2}=1.5$; Parameters: $\gamma^{2}=0.025, \mu_{1}=0.001, \mu_{2}=0.00025, \varepsilon=2, N_{e}=10,000$. 

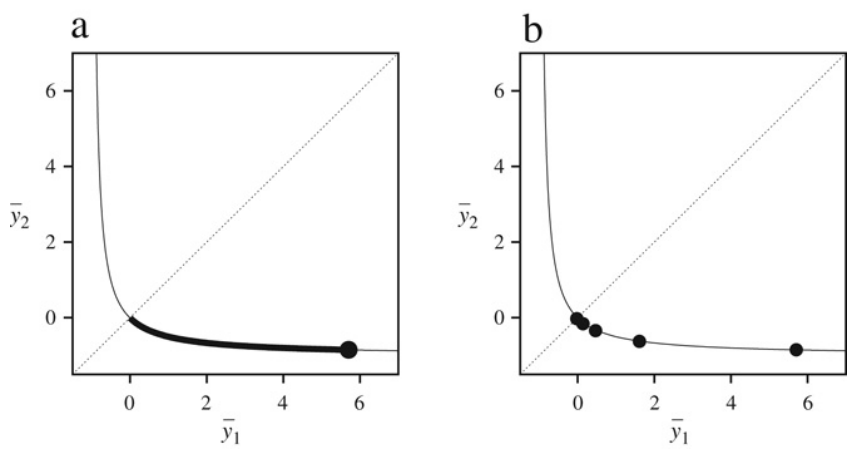

Fig. 7. An illustration of the arc length of a genotype. In (a), the arc length corresponding to an equilibrium point in the $\bar{y}_{1}, \bar{y}_{2}$ plane (thick dot) is marked by the thick line (parameters: $N_{e}=10,000, \gamma^{2}=0.05, \varepsilon=2, u_{1}=0.001$, $u_{2}=0.0001$ ). The thin line is the iso-phenotype contour and the dotted line the main diagonal. (b) shows the predicted equilibrium points for (from right to left) $u_{2}=0.0001,0.00025,0.0005,0.00075,0.001$ and the other parameters the same as in (a). This corresponds to (D2) in Fig. 8.

\subsection{Genetic variance and mean phenotype}

Both our analytical and numerical results confirm the predictions of Hermisson et al. (2003) for the effect of epistasis on the phenotypic mean and the equilibrium genetic variance. In particular, the phenotype mean deviates from the optimal phenotype in the direction of antagonistic epistasis (the shallower slope of the fitness ridge). The genetic variance in the epistatic model is reduced relative to the standard additive one (Fig. 3). In the model with genetic drift, these qualitative predictions are confirmed even for cases where stochasticity and evolutionary inertia are dominant.

All effects of epistasis are usually small second-order corrections. For example, epistasis reduces the genetic variance $V_{G}$ of the additive model by a term proportional to $\varepsilon^{2} V_{G}^{2}$. Larger effects of epistasis are therefore only seen for large $\varepsilon \gg 1$ (see also Fig. A.1 in the Appendix). Since part of the genetic variance $V_{G}=V_{A}+V_{A A}$ is due to epistatic variance $V_{A A}$, which increases proportional to $\varepsilon^{2}$ (Eq. (8)), the reduction in $V_{G}$ is entirely due to a reduction in the additive component $V_{A}$. For very large epistasis, the equilibrium $V_{A}$ in the model tends to zero. The population then crosses over to a different type of equilibrium, where $V_{A}=0$ and $V_{G}=V_{A A}$ increases with further increase of $\varepsilon$ (see the Appendix for details). A vanishing $V_{A}$ is, however, usually not observed in natural traits. Since the number of potential interactions sharply increases with the number of loci, the combined effect of epistasis may be more prominent in a multi-locus model (Hermisson et al., 2003). In any case, since the effect on $V_{G}$ is always negative, epistasis can never be a factor to explain high levels of genetic variance in mutation-selection-drift balance. (In the Appendix this is confirmed also in the presence of additional dominance terms, see Fig. A.2.)

We find that, with epistasis, linkage disequilibria have an even smaller effect on the equilibrium quantities than in the additive model. Even for quite strong linkage $(r \approx 0.001)$ almost no deviations from the predicted linkage equilibrium results are seen. The reason is that disequilibria result from epistasis for fitness, which also exists in the additive model with stabilizing selection. Additional epistasis on the level of the trait introduces an additional degree of freedom that is used by the population to deviate (slightly) from the phenotypic optimum to the flatter and less curved side of the fitness landscape. As a consequence, epistasis for fitness is slightly reduced.

\subsection{Evolution of the genetic architecture}

A direct consequence of gene interactions is that allelic (or mutational) effects at epistatic loci can evolve due to changes in the genetic background. In our minimal two-locus model, the genetic background of each locus is given by the allelic state at the other locus. There is a single degree of freedom for the evolution of the genetic architecture. Fig. 1 shows that the fitness landscape forms a hyperbolic ridge around the isocline of the optimal phenotype. As the population moves along this ridge, the locus effects evolve in opposite directions. Note that, in the absence of higher-order epistasis, the epistasis parameter $\varepsilon$ itself cannot evolve. A complementary model has been studied by Liberman and Feldman $(2006,2007)$, where single-locus effects are fixed, but epistasis between the two loci can evolve due to mutations at a modifier locus.

A classical expectation is that epistatic traits under stabilizing selection should evolve mutational robustness or canalization (Wagner et al., 1997). A canonical measure of the degree of canalization is the mutational variance of a trait. A minimum in $V_{m}$ corresponds to maximal canalization. In the present model, the maximal canalization that can be achieved on the fitness ridge corresponds to the point where both locus components of the mutational variance are equal, $V_{m, 1}=V_{m, 2}$. The main result of Hermisson et al. (2003), however, was that evolution does not maximize canalization on the level of the trait. Instead, strong buffering only evolves at the locus with the higher mutation rate. In particular, for $u_{1}>u_{2}$, the locus effects are predicted to evolve such that the opposite relation holds for the locus-specific mutational variances, i.e. $u_{1} \gamma_{1}^{2}\left\langle f_{1}^{2}\right\rangle<u_{2} \gamma_{2}^{2}\left\langle f_{2}^{2}\right\rangle$. This means that the population is expected to move to a quite eccentric position on the ridge where variation in the locus with larger $u$ keeps the population on the ridge and at high fitness, but variation in the other locus would push it down the slope. The same qualitative prediction follows from the more precise (S)HG formalism above.

To some extent, our numerical results confirm these expectations. In particular, evolution of the genetic architecture does occur and buffering of the locus with the larger mutation rate is the driving force. A quick approach of the fitness ridge is followed by a (slow) movement on the ridge in the predicted direction (Fig. 6). Also the inversion in the order of locus mutational variances is clearly seen and still happens in a time-frame $(\leq 100,000$ generations) of evolutionary relevance (Fig. 10). Nevertheless, there is often no convergence to the equilibrium points even after millions of generations. In particular, if mutation rates are low and differ strongly among loci, we observe a high degree of adaptive inertia, where the population gets almost stuck in its approach of the equilibrium points.

We use the term "adaptive inertia" in the sense of Baatz and Wagner (1997). It refers to a slowing-down of the adaptive process not as a result of weak selection, but because of variational constraints. In the case of Baatz and Wagner (1997), these constraints result from deleterious pleiotropic effects: Their "corridor model" describes selection on two traits, one under directional selection and the other one under stabilizing selection. In a two-dimensional plot spanned by the two traits, the fitness landscape thus forms an ascending ridge. Baatz and Wagner (1997) assume that all mutations have effects on both traits. Since the effects on the trait under stabilizing selection are almost always deleterious (i.e. they lead off the ridge), progress in the adaptation of the trait under directional selection is slowed or even stopped. Also in our model, approach to the equilibrium points is only possible on a narrow fitness ridge with steep slopes. Single mutations tend to push the population off the ridge. In contrast to the corridor model, variational constraints arise from epistasis rather than from pleiotropy. In fact, for progress on the ridge, finetuned changes at both loci are necessary. This becomes difficult, in particular, if the locus with the (anyway) smaller mutation rate (locus 2 in the examples) evolves such that almost all mutations are strongly selected against. 

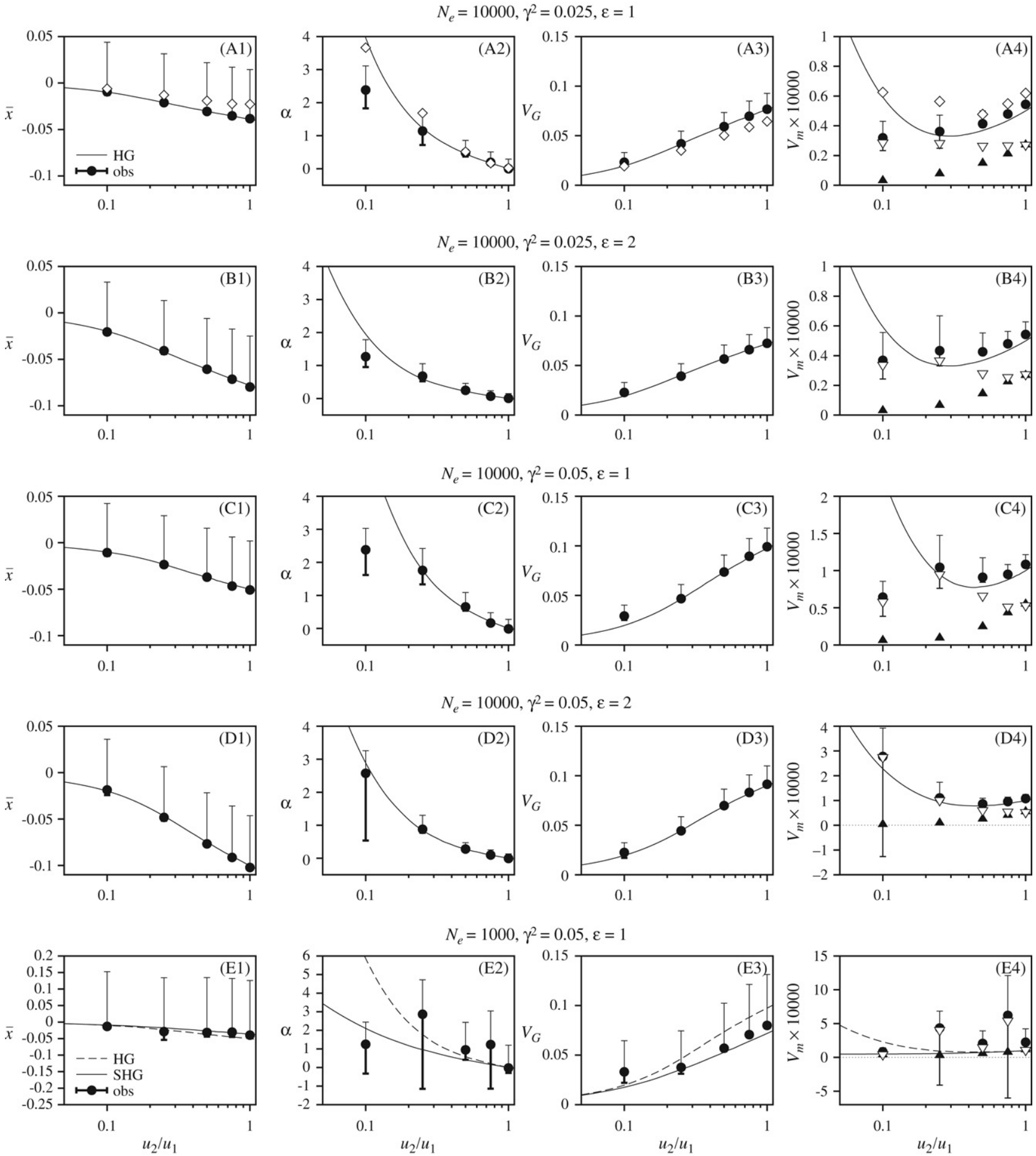

Fig. 8. Mean phenotype $\bar{x}$, arc length $\alpha$, genetic variance $V_{G}$ and mutational variance $V_{m}$ in simulations with unequal loci. $u_{1}=0.001$ is held constant, whereas $u_{2}$ takes values of $0.0001,0.00025,0.0005,0.00075$ and 0.001 (note the logarithmic scale of the horizontal axis). The lower (thick) error bars are the standard deviations of the 10 replicate means. The upper (thin) error bars show the standard deviations over time, averaged over all replicates. For $N_{e}=10,000$, the solid line is the prediction from the HG approximation. For $N_{e}=1000$, the solid line is the prediction from the SHG, and the dotted line the prediction from the HG. In the first row, diamonds show the mean observed values in a model with complete linkage $(r=0)$. In the right-most column, the closed triangles show the mutational variance due to locus 1 , and the open triangles the mutational variance due to locus 2 . Note that the locus with the lower mutation rate (locus 2 ) evolves to have the higher mutational variance.

The analogy to Baatz and Wagner (1997) can be made even closer if we think of the trait and its genetic architecture as of two different traits. There is then an almost necessary pleiotropic relation between both traits. And evolution of an optimal genetic architecture is slowed by deleterious side-effects of the mutations on the trait value itself. Our results show that adaptive inertia then naturally results even in a continuum-of-alleles model.

Adaptive inertia does not itself depend on population size. In smaller populations, however, fixation of sub-optimal mutations at the low-mutation-rate locus due to drift becomes possible. Compensatory evolution at the locus with the larger mutation rate 

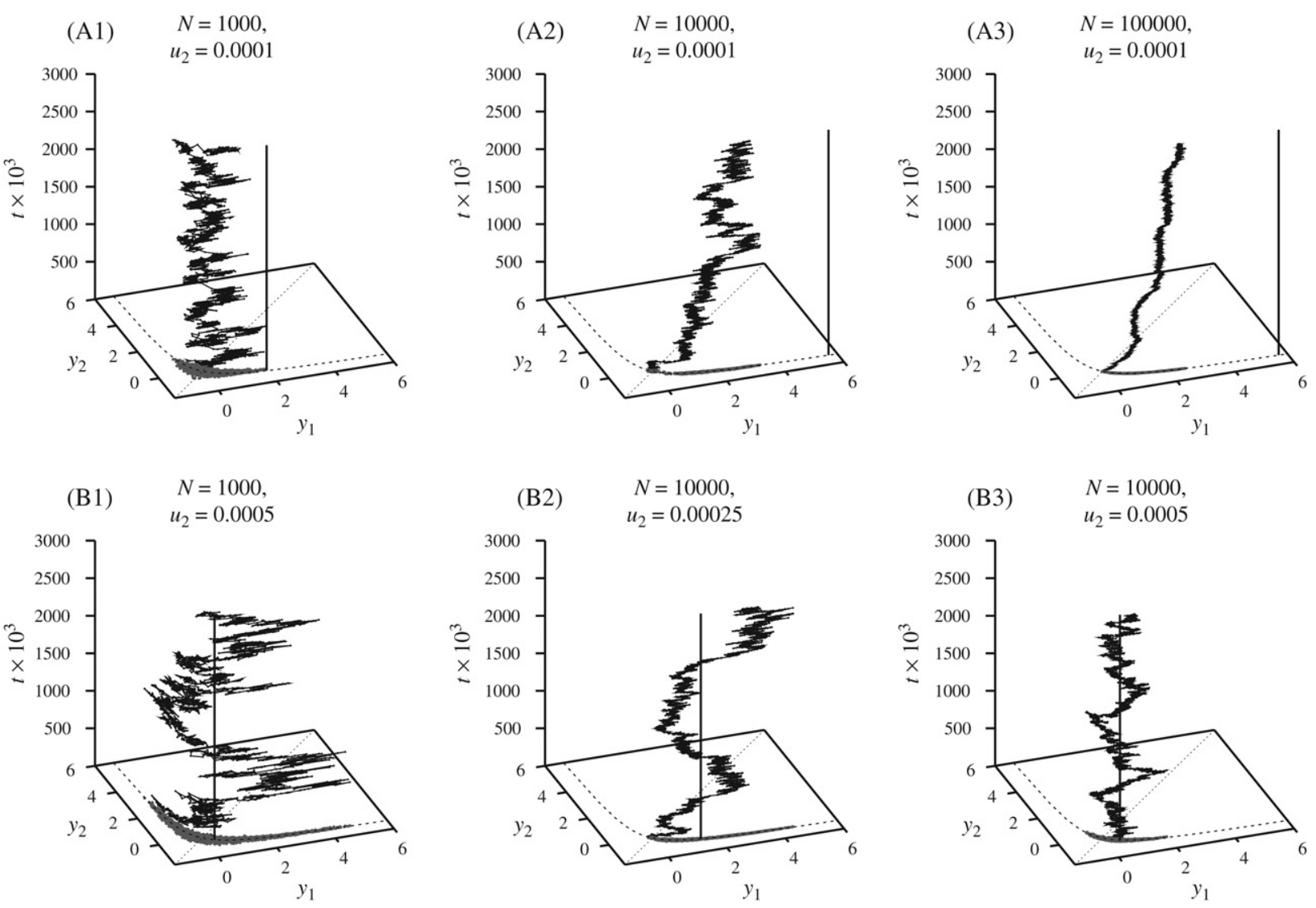

Fig. 9. Examples of the evolutionary trajectory of populations in the $\bar{y}_{1}, \bar{y}_{2}$ plane for $\gamma^{2}=0.05$ and $\varepsilon=1$. The vertical axis is time in 1000 generations. The solid vertical line marks the predicted equilibrium point according to the HG $\left(N_{e} \geq 10,000\right)$ or SHG $\left(N_{e}=1000\right)$ approximation. The dashed line is the corresponding iso-phenotype contour (at this resolution, it is indistinguishable from the $x=0$ contour). The dotted line is the diagonal $\bar{y}_{1}=\bar{y}_{2}$. The gray shading is a projection of the data onto the $t=0$ plane. (A1)-(A3) show "adaptive inertia" for three different population sizes and a small mutation rate at locus 2. (B1) and (B2) demonstrate stochastic fluctuations around (and sometimes far off) the predicted equilibrium, whereas (B3) shows a more regular behavior.

then leads to large jumps of the evolutionary trajectory along the ridge. As a result, we obtain an irregular pattern, where long phases of stasis (dominated by inertia) are punctuated by large stochastic jumps.

Stochasticity and inertia have a much larger effect on the mutational variance than on the genetic variance and the phenotype mean. Fig. 6 shows that evolution to the iso-phenotype contour is very fast. Once the contour is reached, the mean phenotype is almost invariable (apart from small stochastic fluctuations). Also $V_{G}$ has almost reached its equilibrium value once the population is on the contour, although there is a slight and slow decrease as the population evolves along the ridge (not shown). The same result holds for the mutation load $L=s\left(\bar{x}^{2}+V_{G}\right)$.

In sharp contrast, major changes of $V_{m}$ and its locus components occur in this later phase of the adaptive process. Since the mutational variance depends on the precise position of the population on the fitness ridge, it is strongly influenced by adaptive inertia, stochastic jumps, and large-scale fluctuations. This is a consequence of the fact that evolution of the genetic architecture (resp. of $V_{m}$ ) is not itself a target of selection, but only a by-product of selection for optimal $\bar{x}$ and reduced $V_{G}$. Interestingly, the same finding of slow convergence and stochastic behavior holds for the variance in fitness, which (by Eq. (12)) is proportional to $V_{m}$.

Our results show the potential for the evolution of genetic architecture, but also clear limits. On the one hand, there is a robust trend for the evolution of buffering, in particular at loci with higher mutation rates. On the other hand, adaptive evolution of the genetic architecture is slowed by inertia. Although we have analyzed these phenomena in a minimal model of only two loci, they should play a similar role in more general multi-locus models. Indeed, if a trait is under selective constraint (e.g. due to stabilizing selection) evolution of its genetic architecture will often only be possible by compensatory mutations at multiple loci. Adaptive inertia may then be an important factor to limit the evolutionary optimization of the genetic architecture of a quantitative trait.

\section{Acknowledgments}

We acknowledge helpful comments from Thomas Hansen and two anonymous reviewers. Technical support was provided by Dietfried Molter and Walter Schultheiss. The work was made possible with financial support by the Deutsche Forschungsgemeinschaft (DFG) and the Vienna Science and Technology Fund (WWTF). JAC was partially funded by grants from FORMAS and the Swedish Foundation for Strategy Research (SSF) to Örjan Carlborg.

\section{Appendix}

\section{A.1. Calculations for finite population size}

Our formalism in the main text assumes an infinite population size. Following Hermisson and Wagner (2004), we can extend the HG approximation to include genetic drift. For finite populations, we need to consider the averages across replicates in the dynamical Eqs. (15) and (17). To a leading order approximation, we ignore all variances and covariances across replicates, i.e., we set $E\left[V_{i}^{2}\right]=$ $E\left[V_{i}\right]^{2}, E\left[\bar{x} \bar{f}_{i}\right]=E[\bar{x}] E\left[\bar{f}_{i}\right]$, etc. Analogous assumptions are made in the well-known stochastic versions of the Gaussian and 

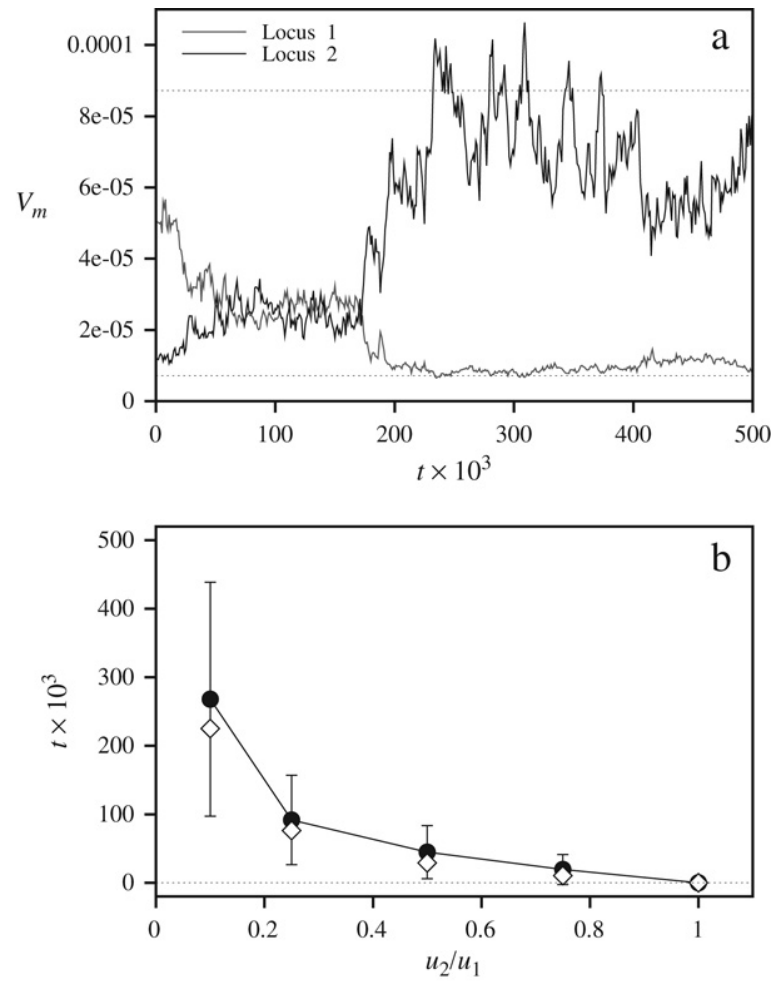

Fig. 10. (a) The dynamics of the per-locus mutational variances $V_{m, i}$ in the first 500,000 generations of an example simulation with $N_{e}=10,000, \gamma^{2}=0.025$ $\varepsilon=1, u_{1}=0.001$, and $u_{2}=0.00025$. The dotted lines are the predictions from the HG approximation. Note the crossing of the variances around generation 100,000 . (b) The time to the first crossing of the per-locus mutational variances, for $u_{1}=0.001$, different values of $u_{2} / u_{1}$ and the other parameters as in (a). Data are means (filled circles), standard deviations (error bars) and medians (diamonds) from 100 replicated simulation runs.

HC approximations (e.g. Bürger (2000, p. 269)). For notational simplicity, we denote replicate averages again by $V_{i} \equiv E\left[V_{i}\right]$, etc. Since genetic drift has no directional effect on the locus means, we then recover the eigenvalue equation (18) in mutation-selectiondrift equilibrium. Since drift removes genetic variance from a population, it enters the dynamical equation for the variance (17). If $N_{e}$ is the diploid variance-effective population size $\left(2 N_{e}\right.$ number of haploids), this effect can be approximated by an additional term $-V / 2 N_{e}$ (Bürger, 2000). We can then define a stochastic version of the HG approximation for the epistatic model as

$E\left[\Delta V_{i}\right] \approx-s\left(V_{i}^{2}+V_{i} \gamma_{i}^{2}\right)\left\langle f_{i}^{2}\right\rangle+u_{i} \gamma_{i}^{2}-\frac{V_{i}}{2 N_{e}}$.

In mutation-selection-drift equilibrium, $E\left[\Delta V_{i}\right]=0$, this leads to the "Stochastic House-of-Gauss" (SHG) approximation for $V_{i}$,

$V_{i}=\frac{\gamma_{i}^{2}}{2 G_{i}\left\langle f_{i}^{2}\right\rangle}\left(\sqrt{G_{i}^{2}\left\langle f_{i}^{2}\right\rangle^{2}+2\left(1+\Theta_{i}\right) G_{i}\left\langle f_{i}^{2}\right\rangle+1}-G_{i}\left\langle f_{i}^{2}\right\rangle-1\right)$,

where $\Theta_{i}=4 N_{e} u_{i}$ is the standard population mutation parameter and $G_{i}=2 N_{e} s \gamma_{i}^{2}=2 N_{e} g_{i}$ measures the selection strength. Like in Eq. (21), the selection strength at each locus, $G_{i}\left\langle f_{i}^{2}\right\rangle$, depends on the epistatic effect of the genetic background (here: the other locus), which is captured by $\left\langle f_{i}^{2}\right\rangle$. We then obtain from Eqs. (23) and (A.2)

$$
\begin{aligned}
V_{1}= & \frac{\gamma_{1}^{2} z}{2 \sqrt{G_{1} G_{2}}}\left(\sqrt{G_{1} G_{2} z^{-2}+2\left(1+\Theta_{1}\right) \sqrt{G_{1} G_{2}} z^{-1}+1}\right. \\
& \left.-\sqrt{G_{1} G_{2}} z^{-1}-1\right)
\end{aligned}
$$

and for $V_{2}$ the same equation with $\gamma_{1}^{2}$ and $\Theta_{1}$ replaced by $\gamma_{2}^{2}$ and $\Theta_{2}$, and $z$ replaced by $z^{-1}$. Using Eq. (24), we can again express $\sqrt{V_{1} V_{2}}$ in two ways as

$$
\begin{aligned}
\sqrt{V_{1} V_{2}}= & \frac{1}{4 N_{e} s}\left(\sqrt{\left(\sqrt{G_{1} G_{2}} z+1\right)^{2}+2 \Theta_{2} \sqrt{G_{1} G_{2}} z}\right. \\
& \left.-\sqrt{G_{1} G_{2}} z-1\right) \\
= & \frac{1}{4 N_{e} s}\left(\sqrt{\left(\sqrt{G_{1} G_{2}} z^{-1}+1\right)^{2}+2 \Theta_{1} \sqrt{G_{1} G_{2}} z^{-1}}\right. \\
& \left.-\sqrt{G_{1} G_{2}} z^{-1}-1\right) .
\end{aligned}
$$

and we obtain the following equation for $z$,

$$
\begin{gathered}
\Theta_{1}\left(\Theta_{1}+2\right) z^{-2}+\Theta_{2}\left(\Theta_{2}+2\right) z^{2}-2 \sqrt{G_{1} G_{2}}\left(\Theta_{1}-\Theta_{2}\right) \\
\left(z-z^{-1}\right)-2\left(\Theta_{1}+\Theta_{2}+\Theta_{1} \Theta_{2}\right)=0 .
\end{gathered}
$$

Note that the infinite-size limit (28) can be reproduced from (A.6) by considering only the leading order terms proportional to $N_{e}^{2}$.

\section{A.2. Calculation of arc lengths}

In the following, we describe how to calculate the arc lengths. Consider a population on the epistatic landscape. Its position on the landscape is described by the average locus effects (reference effects) $\bar{y}_{1}$ and $\bar{y}_{2}$. Due to the selective constraint, evolution (and stochastic fluctuations) of the population mostly occur along the fitness ridge, which is a curved (hyperbolic) line in the $\left(\bar{y}_{1}, \bar{y}_{2}\right)$ plane. As a consequence, averages over replicates or across generations in the $\bar{y}_{i}$ will introduce a bias and an apparent shift of the population off the fitness ridge. An alternative parametrization that avoids this problem is to use the mean phenotype $\bar{x}$ and the arc-length $\alpha$ along the iso-phenotype contour. The iso-phenotype contour that corresponds to $\bar{x}$ is defined by the formula

$y_{2}=\frac{\bar{x}-y_{1}}{1+\varepsilon y_{1}}$.

The intersection of this contour with the main diagonal $y_{1}=y_{2}$ is at $y_{1}=y_{2}=(\sqrt{1+\varepsilon \bar{x}}-1) / \varepsilon$. The arc length $\alpha$ from this point to the point $\left(\bar{y}_{1}, \bar{y}_{2}\right)$ is given by

$\alpha\left(\bar{y}_{1}, \bar{y}_{2}\right)=\int_{\frac{\sqrt{1+\varepsilon \bar{x}}-1}{\varepsilon}}^{\bar{y}_{1}} \sqrt{1+\frac{(1+\varepsilon \bar{x})^{2}}{\left(1+\varepsilon y_{1}\right)^{4}}} \mathrm{~d} y_{1}$,

where we use the linkage-equilibrium value of the mean phenotype, $\bar{x}=\bar{y}_{1}+\bar{y}_{2}+\varepsilon \bar{y}_{1} \bar{y}_{2}$.

\section{A.3. Large epistasis}

The HG approximation gives an accurate approximation for the phenotype mean $\bar{x}$ and the genetic variance $V_{G}$ over a wide range of parameter values. In particular, the influence of the strength of epistasis is shown in Fig. A.1. With increasing epistasis, the population deviates ever more strongly from the optimal phenotype contour in the direction of the flatter slope of the fitness landscape. As a consequence, the deviation of the mean phenotype from the optimum, $|\bar{x}|$, increases and the genetic variance $V_{G}$ decreases. For very strong epistasis $(\varepsilon=8$ and $\varepsilon=16$ in Fig. A.1), however, this trend is limited or even reversed. The reason is a peculiarity of the model that has already been discussed by Hermisson et al. (2003). It is easy to see that the phenotype (and fitness) landscape of the model is point symmetric with respect to $\left(y_{1}, y_{2}\right)=(-1 / \varepsilon,-1 / \varepsilon)$. At this point the landscape is completely flat in the direction of both loci, we have $f_{1}=f_{2}=$ 0 and $x=-1 / \varepsilon$. Once the mean phenotype of the population has reached this point, it will not move any further even for 
(A1) $\quad u=0.0001$

(B1)
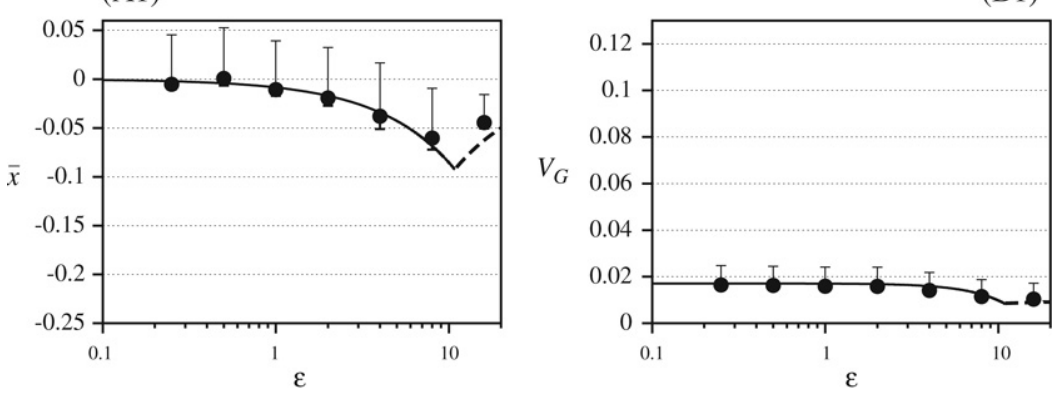

(A2)

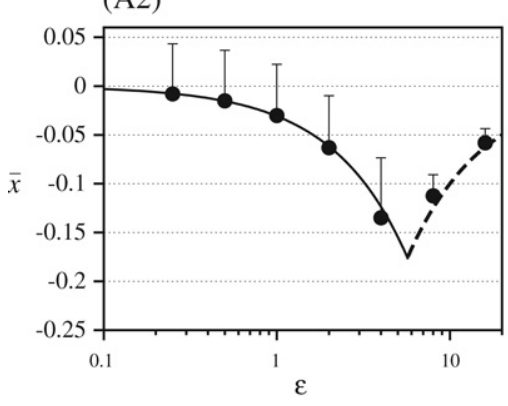

(A3)

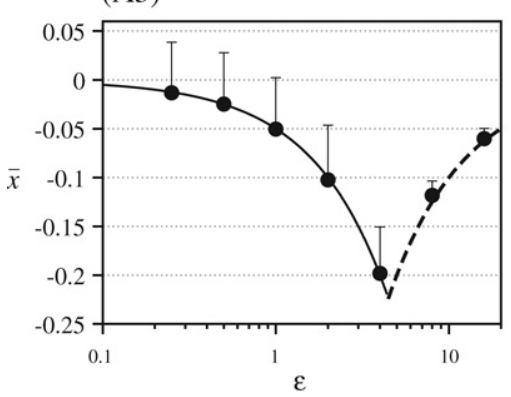

$u=0.0005$

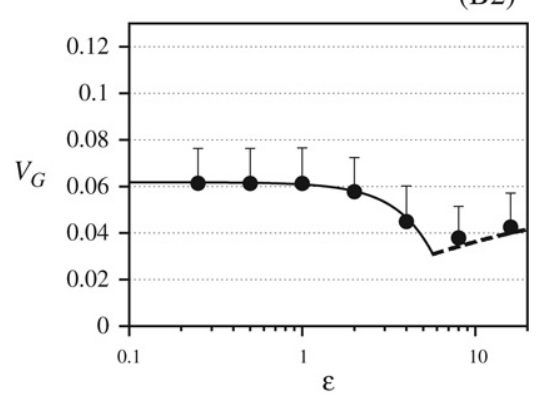

$u=0.001$

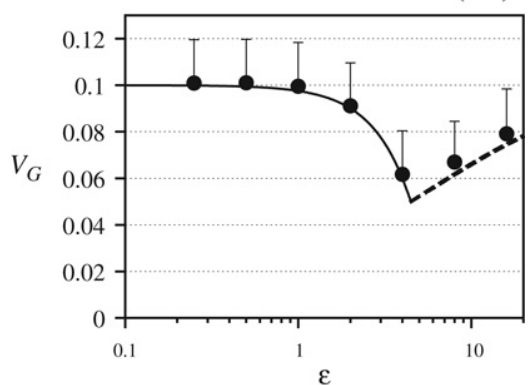

Fig. A.1. Equilibrium mean phenotype $\bar{x}$ and genetic variance $V_{G}$ as a function of the epistasis parameter $\varepsilon$. Parameters at both loci are equal, with $\gamma^{2}=0.05$ and mutation rate as indicated. The population size is $N_{e}=10,000$. Error bars are as in Fig. 2. The solid line is the prediction for the HG approximation. For large epistasis, transition to the $V_{A}=0$ equilibrium occurs (dashed line; see Appendix text for details).

stronger epistasis. In a flat landscape, the additive component $V_{A}$ of the genetic variance is zero, i.e. all genetic variance stems from the epistatic component. Formally, the population crosses over to a different stable equilibrium, called $V_{A}=0$ equilibrium in Hermisson et al. (2003). The HG predictions for this equilibrium can easily be derived. Assume equal loci, for simplicity. From $\bar{f}=0$, we then obtain $\bar{x}=-1 / \varepsilon$ from (7) and $\left\langle f^{2}\right\rangle=\varepsilon^{2} V$ from (10). Inserting $\left\langle f^{2}\right\rangle$ into (21), we can solve for $V$, yielding

$V=\frac{\gamma^{2}}{4}\left(\sqrt{1+\frac{8 u}{s \gamma^{2}}}-1\right)$

and obtain $V_{G}$ (and everything else) from this solution. The predictions are included as dashed lines in Fig. A.1. They produce a reasonable fit with the simulation data. Since natural traits usually do not have a vanishing additive genetic variance, this parameter regime may be of little practical relevance, however.

\section{A.4. Dominance}

We can extend the model to include interactions within a locus, or dominance, by adding terms proportional to $y_{1}^{2}$ and $y_{2}^{2}$,

$x=x_{\mathrm{opt}}+y_{1}+y_{2}+\varepsilon y_{1} y_{2}+\delta\left(y_{1}^{2}+y_{2}^{2}\right)$.
It turns out that this model is no longer analytically tractable within our framework, even in the absence of epistasis, $\varepsilon=0$. The formal reason is that the equilibrium distribution at both loci is no longer symmetric. Therefore, the odd cumulants of third and higher order no longer vanish in linkage equilibrium. To check the stability of our results to the inclusion of dominance, we have performed limited additional simulations. As shown in Fig. A.2, the equilibrium genetic variance is most strongly affected for large mutation rates and positive values of the dominance parameter $\delta$. In contrast, negative values of $\delta$ have at most a slight effect. Note that the simulation results support our general observation that gene interactions tend to reduce the equilibrium variance relative to the additive case. On a global scale, inclusion of quadratic dominance terms leads to strong changes of the phenotype and fitness landscape. For example, if the dominance parameter is $\delta=$ 1 , the iso-phenotype contours (and hence the fitness ridge) are no longer curved, but are straight lines parallel with $y_{2}=-y_{1}$. Since the fitness landscape is translationally invariant in this direction, there is also no selective force to drive the population along the ridge. Our results for adaptive inertia can no longer apply in such a case. In contrast, the curvature of phenotype and fitness contours becomes stronger for negative $\delta$. 


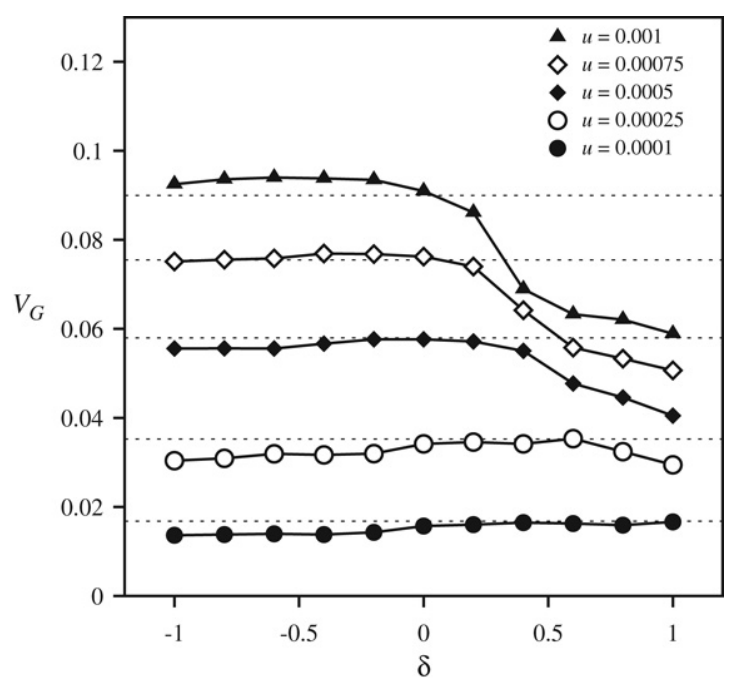

Fig. A.2. The effect of "dominance" in the model with identical loci and epistasis The plots show the genetic variance $V_{G}$ as a function of the dominance parameter $\delta$ for various values of the the per-locus mutation rate $u$ in a large population $\left(N_{e}=10,000\right)$ for $\varepsilon=2$ and $\gamma^{2}=0.05$ (cf., Fig. 3B1-B3). Note that error bars for the mean standard deviation over time are not shown. Error bars for the standard deviation of replicate means (corresponding to lower error bars in Figs. 2, 3 and 8 ) are too small to be visible. The horizontal dotted lines show the HG predictions for $V_{G}$ at $\delta=0$.

\section{References}

Álvarez-Castro, J.-M., Carlborg, O., 2007. A unified model for functional and statistical epistasis and its application in quantitative trait loci analysis. Genetics 176, 1151-1167.

Baatz, M., Wagner, G.P., 1997. Adaptive inertia caused by hidden pleiotropic effects. Theoret. Popul. Biol. 51, 49-66.

Barton, N.H., Turelli, M., 2004. Effects of genetic drift on variance components under a general model of epistasis. Evolution 58, 2111-2132.

Bürger, R., 2000. The Mathematical Theory of Selection, Recombination, and Mutation. Wiley, Chichester.

Carter, A.J.R., Hermisson, J., Hansen, T.F., 2005. The role of epistatic gene interactions in the response to selection and the evolution of evolvability. Theoret. Popul. Biol. 68, 179-196.

Cheverud, J., Routman, E., 1995. Epistasis and its contribution to genetic variance components. Genetics 139, 1455-1461. de Visser, J.A.G.M., Hermisson, J., Wagner, G.P., Ancel Meyers, L., Bagheri-Chaichian, H., Blanchard, J.L., Chao, L., Cheverud, J.M., Elena, S.F., Fontana, W., Gibson, G., Hansen, T.F., Krakauer, D., Lewontin, R.C., Ofria, C., Rice, S.H., von Dassow, G., Wagner, A., Whitlock, M.C., 2003. Perspective: Evolution and detection of genetic robustness. Evolution 57, 1959-1972.

Fisher, R.A., 1918. The correlation between relatives on the supposition of Mendelian inheritance. Trans. Royal Soc. Edinburgh 52, 399-433.

Flatt, T., 2005. The evolutionary genetics of canalization. Quat. Rev. Biol. 80, 287-316.

Hansen, T.F., 2006. The evolution of genetic architecture. Ann. Rev. Ecol. Evol. Syst. 37, 123-157.

Hansen, T.F., Álvarez-Castro, J.-M., Carter, A.J.R., Hermisson, J., Wagner, G.P., 2006. Evolution of genetic architecture under directional selection. Evolution 60, 1523-1536.

Hansen, T.F., Wagner, G.P., 2001. Modeling genetic architecture: A multilinear theory of gene interaction. Theoret. Popul. Biol. 59, 61-86.

Hermisson, J., Hansen, T.F., Wagner, G.P., 2003. Epistasis in polygenic traits and the evolution of genetic architecture under stabilizing selection. Amer. Nat. 161, 708-734.

Hermisson, J., Wagner, G.P., 2004. The population genetic theory of hidden variation and genetic robustness. Genetics 168, 2271-2284.

Kao, C.H., Zeng, Z.B., 2002. Modeling epistasis of quantitative trait loci using Cockerham's model. Genetics 160, 1243-1261.

Kopp, M., Hermisson, J., 2006. The evolution of genetic architecture under frequency-dependent disruptive selection. Evolution 258, 1537-1550.

Lande, R., 1976a. The maintenance of genetic variability by mutation in a polygenic character with linked loci. Gen. Res. Camb. 26, 221-235.

Lande, R., 1976b. Natural selection and random genetic drift in phenotypic evolution. Evolution 30, 314-334.

Liberman, U., Feldman, M.W., 2006. Evolutionary theory or modifiers of epistasis using a general symmetric model. Proc. Natl. Acad. Sci. USA 103, 19402-19406.

Liberman, U., Feldman, M.W., 2007. On the evolution of epistasis III: The haploid case with mutation. Theoret. Popul. Biol. 73, 307-316.

Rice, S.H., 1998. The evolution of canalization and the breaking of von Baer's laws: Modeling the evolution of development with epistasis. Evolution 52, 647-656.

Rice, S.H., 2002. A general population genetic theory for the evolution of developmental interactions. Proc. Natl. Acad. Sci. USA 99, 15518-15523.

Turelli, M., 1984. Heritable genetic variation via mutation-selection balance: Lerch's zeta meets the abdominal bristle. Theoret. Popul. Biol. 25, 138-193.

Turelli, M., Barton, N., 1990. Dynamics of polygenic characters under selection. Theoret. Popul. Biol. 38, 1-57.

Waddington, C.H., 1953. The genetic assimilation of an aquired character. Evolution 7, 118-126.

Wagner, G.P., Booth, G., Bagheri-Chaichian, H., 1997. A population genetic theory of canalization. Evolution 51, 329-347.

Waxman, D., 2003. Numerical and exact solutions for continuum of alleles models. J. Math. Biol. 46, 225-240.

Wolf, J.B., Brodie III, E.D., Wade, M.J., 2000. Epistasis and the Evolutionary Process. Oxford University Press, Oxford.

Yang, R.C., 2004. Epistasis of quantitative trait loci under different gene action models. Genetics 167, 1493-1505.

Zeng, Z.B., Wang, T., Zou, W., 2005. Modeling quantitative trait loci and interpretation of models. Genetics 169, 1711-1725. 